\title{
Risk measures and capital requirements for processes
}

\author{
Marco Frittelli, Giacomo Scandolo \\ Università degli Studi di Firenze, Italy \\ MATHEMATICAL FINANCE* 2006, Vol. 16/4, pp. 589-612.
}

\begin{abstract}
In this paper we propose a generalization of the concepts of convex and coherent risk measures to a multi-period setting, in which payoffs are spread over different dates. To this end, a careful examination of the axiom of translation invariance and the related concept of capital requirement in the one-period model is performed. These two issues are then suitably extended to the multi-period case, in a way that makes their operative financial meaning clear. A characterization in terms of expected values is derived for this class of risk measures and some examples are presented.
\end{abstract}

\section{Introduction}

Recently, a growing literature has been devoted to an axiomatic theory of risk measures. In particular, a set of four desirable properties that every sound or, better, coherent risk measure should satisfy has been proposed by Artzner et al. (1999) and a representation result in terms of expected values has been derived under a mild continuity assumption by Delbaen (2000). Subsequently, Föllmer and Schied (2002) and independently Frittelli and Rosazza Gianin (2002) broadened the class of coherent risk measures, defining convex risk measures and extending to them Delbaen's representation result. The setting of this theory is commonly said to be static, since the financial positions are described by random variables and no temporal dimension is involved in the risk measurement.

The scope of this paper is to generalize this theory to a dynamic setting, in which the financial positions are described by discrete-time stochastic processes. The reason for this extension is dictated by the undeniable fact that in current financial practice risky payoffs are usually spread over different dates. Moreover, the temporal order of the various subsequent payoffs is relevant both on a psychological and an operative level. This aspect seems to be neglected by a common methodology to measure riskiness of payoff streams, namely the application of a static risk measure to the sum of all properly discounted payoffs. We will deal with this issue in Section 6 . We assume throughout the paper that every future amount is properly discounted, so that interest rates will not formally enter our modeling.

Some earlier papers, such as Artzner et al. (2001), Pflug (2001), Riedel (2002) and Weber (2004) have addressed this extension at different degrees of generality and within different financial interpretations of the processes. All these papers extend in a common, natural way three out of the four coherency axioms proposed by Artzner et al (1999) in the static setting,

\footnotetext{
${ }^{*}$ Manuscript received March2004; final version received July 2005.
} 
namely convexity, monotonicity and positive homogeneity. However, they propose different generalizations of the fourth axiom, that of translation invariance, which in the static setting states that

$$
\rho(X+\alpha)=\rho(X)-\alpha \quad \forall X \in L, \alpha \in \mathbb{R},
$$

where $L$ is a space of random variables and $\rho: L \rightarrow \mathbb{R}$ is a risk measure.

In this paper we take quite a different route since, rather than proposing a new definition of translation invariance, we introduce a general functional form for a risk measure, which we call a capital requirement and which can be applied to a number of different cases. Here, we briefly explain the main idea, referring to Section 3 for a rigorous formulation. It is easy to observe that any map $\rho: L \rightarrow \mathbb{R}$ (interpreted as a static risk measure) satisfies (TI) if and only if it is a simple capital requirement, i.e.

$$
\rho(X)=\rho_{\mathcal{A}}(X) \triangleq \inf \{\alpha \in \mathbb{R} \mid X+\alpha \in \mathcal{A}\},
$$

for some set $\mathcal{A} \subset L$. From the operative viewpoint, the definition of $\rho_{\mathcal{A}}$ can be interpreted as follows. Once the set $\mathcal{A}$, collecting all acceptable positions, has been fixed, the agent is naturally interested in the minimal sum $\alpha$ which has to be set aside, maybe invested in a risk-free account, in order that the overall final position $X+\alpha$ is acceptable. Formally, we can also write:

$$
\rho_{\mathcal{A}}(X)=\rho_{\mathcal{A}, \mathcal{C}, \pi}(X) \triangleq \inf \{\pi(Y) \in \mathbb{R} \mid Y \in \mathcal{C}, X+Y \in \mathcal{A}\},
$$

where $\mathcal{C}=\mathbb{R} \subset L$ and $\pi: \mathcal{C} \rightarrow \mathbb{R}$ is defined by $\pi(\alpha)=\alpha$. Broadly speaking, the set $\mathcal{C}$ collects all positions achievable by means of permitted hedging strategies and $\pi(Y) \in \mathbb{R}$ is the initial cost of a particular element $Y \in \mathcal{C}$. Observe that the financial meaning of the map $\rho_{\mathcal{A}, \mathcal{C}, \pi}$ is clear even if we allow for different specifications of the set $\mathcal{C}$ and the map $\pi$. For instance, the agent could be allowed to trade on an underlying market. In this case $\mathcal{C}$ could be the set of all replicable claims and $\pi$ the (unique) no-arbitrage pricing rule on $\mathcal{C}$; in particular, if $\mathcal{A}$ is the cone of non-negative random variables, then $\rho_{\mathcal{A}, \mathcal{C}, \pi}(-X)$ coincides with the super-replication price of the claim $X$. Moreover, this scheme can be applied to different objects, other than random variables. Indeed, in this paper we will be mainly interested in the case when $L$ is a space of processes. Whatever the financial objects described by the space $L$ are, the map $\rho_{\mathcal{A}, \mathcal{C}, \pi}$ which we call the capital requirement associated to $\mathcal{A}, \mathcal{C}$ and $\pi$, provides a possible operative quantification of risk.

In connection with the notion of capital requirement formulated in $(\mathrm{CR})$, we address the following specific issues, first at an abstract level and then applying the results to a space of bounded processes which we are most interested in:

1. Properties. In the static setting it is known that properties on the acceptability set $\mathcal{A}$ yield (coherency) properties on the associated simple capital requirement $\rho_{\mathcal{A}}$. For instance, convexity of $\mathcal{A}$ ensures convexity of the map $\rho_{\mathcal{A}}$. In Section 3 , under suitable assumptions on $\pi$ and $\mathcal{C}$, we extend this result to general capital requirements. In the multiperiod setting (see Section 4) we also introduce simple sufficient conditions ensuring that the map $\rho_{\mathcal{A}, \mathcal{C}, \pi}$ is finite-valued, and we discuss their financial meaning.

2. Translation invariance. As we mentioned, in the static setting the property of translation invariance fully characterizes the functional form of a simple capital requirement. In the general setting, this is no more true. However, we show that, under a suitable condition, an appropriate modification of the translation invariance property (Definition 3.4) fully characterizes general capital requirements (see Propositions 3.6 and 4.10). 
3. Representation. If a simple capital requirement $\rho_{\mathcal{A}}$ is convex and monotone -i.e. it is a convex risk measure - then, under a proper continuity condition, it can be written as

$$
\rho_{\mathcal{A}}(X)=\sup _{Q \ll P}\left\{-E_{Q} X-F(Q)\right\}
$$

where $F(Q) \triangleq \sup _{X \in \mathcal{A}}\left\{-E_{Q} X\right\} \in \mathbb{R} \cup\{+\infty\}$. The representation (1.1) is essentially due to the convexity property (see Frittelli and Rosazza Gianin (2002)), but the presence of the normalized dual functional (i.e. the representation in terms of expected values, where $\left.E_{Q} 1=1\right)$ depends on the property (TI) and therefore cannot be extended to the dynamic setting in an direct way. We will derive a similar, but much more general result for general capital requirements (see Proposition 3.9). In particular, when $L$ is a space of bounded processes, the expected values $E_{Q} X$ are possibly weighted by some constants $c \in(0,1]$, and $F$ contains a second term, depending on $\pi$ and $\mathcal{C}$, which vanishes in the static setting, when $\mathcal{C}=\mathbb{R}$ and $\pi=i d$.

4. Credit constraint and the relevance of the payoffs temporal order. In the framework of processes, we present a relevant example of a capital requirement in which, through a careful specification of $\mathcal{C}$, a credit line is imposed in the agent's hedging activity. This capital requirement will have the following representation (see Section 6):

$$
\rho_{\mathcal{A}, \mathcal{C}, \pi}(\mathbf{X})=\sup _{(\mathbf{c}, \mathbf{Q}) \in \mathcal{B}}\left\{-\sum_{n=1}^{N} c_{n} E_{Q_{n}} X_{n}-G(\mathbf{c}, \mathbf{Q})\right\}
$$

where $\mathbf{X}=\left(X_{1}, \ldots, X_{N}\right)$ is the payoff stream; $X_{n}$ is the random payoff at time $t_{n} ; G$ is a penalty function and $\mathcal{B} \triangleq\left\{\left(c_{n}, Q_{n}\right)_{n=1}^{N} \mid c_{n} \in \mathbb{R}_{+}, Q_{n}\right.$ is a $P$ - a.c. probability on $\left.\left(\Omega, \mathcal{F}_{n}\right)\right\}$. In this example we clarify the relevance of the order of the payoffs $X_{n}$ when assessing the risk of $\mathbf{X}$ and show that this feature is due to the presence of the decreasing sequence $\left(c_{n}\right) \in(0,1]$, which we allow to be different from the sequence constantly equal to 1 . This can be useful to treat and study capital requirements for which $\rho_{\mathcal{A}, \mathcal{C}, \pi}(\mathbf{X})>\rho_{\mathcal{A}, \mathcal{C}, \pi}(\mathbf{Y})$ when $\mathbf{X}=\left(-1,1, X_{3}, \ldots, X_{N}\right)$ and $\mathbf{Y}=\left(1,-1, X_{3}, \ldots, X_{N}\right)$ (to drastically simplify, one can also take $X_{n}=0$ for all $\left.n \geq 3\right)$. Recall that we are always assuming zero interest rate, so that the previous phenomenon can only be ascribed to temporal risk aversion.

The paper is structured as follows. In Section 2 we briefly review the main definitions and results about convex risk measures and simple capital requirements. Particular emphasis is given to the interpretation of translation invariant maps as capital requirements. Moreover, a new result concerning utility-based risk measures is presented. In Section 3 we develop, in the abstract setting of locally convex topological vector spaces, the mathematical notion of capital requirement. In Section 4 we specialize the results of Section 3 to discrete-time processes. In Sections 5 we will present an example of a capital requirement under a constraint on the decision timing of the agent's hedging activity and Section 6 is devoted to the discussion of the credit constraint example sketched above. Finally, in Section 7 we show how to construct the set $\mathcal{A}$ when dealing with processes and propose some concrete examples. 


\section{The one-period setting}

\subsection{Coherency axioms}

Let $T>0$ be a fixed future date and $(\Omega, \mathcal{F}, P)$ a probability space, where $\mathcal{F}$ is interpreted as the information available in $T$. The space $L^{\infty}=L^{\infty}(\Omega, \mathcal{F}, P)$ collects all random variables, modeling a (bounded) financial position. With this term, one usually means: (1) a net payoff or (2) a portfolio market value in $T$ or even (3) the change of this value between 0 and $T$. Positive values of $X \in L^{\infty}$ correspond to (relative) gains, while negative values correspond to (relative) losses.

On a general level, a risk measure is any map $\rho: L^{\infty} \rightarrow \mathbb{R}$. Artzner et al. (1999) proposed four axioms that every sound or, in their terminology, coherent risk measure should satisfy. They are:

1. Monotonicity: $X \geq Y$ implies $\rho(X) \leq \rho(Y)$.

2. Convexity: $\rho(\lambda X+(1-\lambda) Y) \leq \lambda \rho(X)+(1-\lambda) \rho(Y)$ for any $X, Y \in L^{\infty}$ and $\lambda \in[0,1]$.

3. Positive homogeneity: $\rho(\lambda X)=\lambda \rho(X)$ for any $X \in L^{\infty}$ and $\lambda \geq 0$.

4. Translation invariance: $\rho(X+\alpha)=\rho(X)-\alpha$ for any $X \in L^{\infty}$ and $\alpha \in \mathbb{R}$.

Remark 2.1 Actually, in the original formulation of the four axioms in Artzner et al., convexity is replaced by subadditivity, i.e. $\rho(X+Y) \leq \rho(X)+\rho(Y)$ for any $X, Y \in L^{\infty}$. However it can be easily proved that these two properties are equivalent under homogeneity (see Frittelli and Rosazza Gianin 2002).

The economic rationale behind monotonicity and convexity is clear. The assumption of homogeneity, instead, is debatable from the financial viewpoint, since homogeneous risk measures seem to fail to detect liquidity risk. Föllmer and Schied (2002) and, independently, Frittelli and Rosazza Gianin (2002) defined the broader notion of convex risk measure, by dropping the hypothesis of homogeneity, while keeping the other three.

\subsection{Simple capital requirements}

The translation invariance axiom identifies coherent/convex risk measures as a subset of an important class of risk measures which have a clear operative meaning: that of capital requirements. Broadly speaking, a capital requirement is the minimal amount $\alpha$ which has to be invested in a risk-free instrument, in such a way that the modified final position $X+\alpha$ is acceptable. Plainly, the practical interpretation of this scheme relies on the nature of $X$ (payoff, market value, etc.). A precise formulation can be given as follows.

A non-empty subset $\mathcal{A} \subset L^{\infty}$, collecting all acceptable or sustainable future positions, is fixed by the agent or by an external regulator and it is called the acceptability set.

Definition 2.2 Let $\mathcal{A}$ be an acceptability set. The quantity

$$
\rho_{\mathcal{A}}(X) \triangleq \inf \{\alpha \in \mathbb{R} \mid X+\alpha \in \mathcal{A}\},
$$

provided it is finite for any $X \in L^{\infty}$, defines a risk measure called the simple capital requirement associated to $\mathcal{A}$. 
The following result, which was essentially pointed out by Föllmer and Schied (2002), is immediate to prove, but it is conceptually crucial in comprehending translation invariance.

Proposition 2.3 A risk measure $\rho$ is translation invariant if and only if it is a simple capital requirement, i.e. $\rho=\rho_{\mathcal{A}}$ for some $\mathcal{A} \subset L^{\infty}$.

Proof. See, for instance, Propositions 4.6 and 4.7 in Föllmer and Schied (2004).

Definition 2.4 A set $\mathcal{A}$ is monotone if $X \geq Y \in \mathcal{A}$ implies $X \in \mathcal{A}$. It is a positive cone if $X \in \mathcal{A}$ implies $\lambda X \in \mathcal{A}$ for any $\lambda \geq 0$.

As $\mathcal{A}$ collects the acceptable positions, then it is natural to assume that it is monotone and convex. It can also be a positive cone, but this further assumption is questionable, as it was the positively homogeneity property of $\rho$.

Proposition 2.5 If $\mathcal{A}$ is monotone (resp. convex, resp. a positive cone, resp. $\sigma\left(L^{\infty}, L^{1}\right)$ closed), then $\rho_{\mathcal{A}}$, provided it is finite, is monotone (resp. convex, resp. pos. homogeneous, resp. $\sigma\left(L^{\infty}, L^{1}\right)$ lower semicontinuous). If $\mathcal{A}$ is monotone, convex and $\sigma\left(L^{\infty}, L^{1}\right)$ closed then $\rho_{\mathcal{A}}$ admits the representation (1.1).

Proof. See Proposition 4.7 and Theorem 4.31 in Föllmer and Schied (2004).

Remark 2.6 It is not difficult to prove that $\rho_{\mathcal{A}}$ is finite valued whenever $\mathcal{A}$ is monotone and satisfies

$$
\exists \gamma \in \mathbb{R} \text { such that } X \leq \gamma \text { implies } X \notin \mathcal{A} \text {. }
$$

Compare this last property with the more general Assumption B in Section 4.3.

Remark 2.7 Plainly it is possible to define coherent/convex risk measures and capital requirements on spaces of possibly unbounded random variables, such as $L^{p}(\Omega, \mathcal{F}, P)$ for $p \in[1,+\infty]$ or even in $L^{0}(\Omega, \mathcal{F}, P)$, the space of all finite valued random variables. The results above hold as well. However we remind that the space $L^{p}(P)$, when $p \in[1,+\infty)$, strictly depends on the probabilistic model $P$, contrary to $L^{\infty}$ and $L^{0}$, and this can be seen as a flaw. Moreover Delbaen (2000) proved that there are no coherent risk measures on $L^{0}$, whenever the probability space is non-atomic. More recently, Cheridito et al (2004b) extended this negative result to convex risk measures.

\subsection{Examples of simple capital requirements}

We provide here some examples of acceptability sets and capital requirements that have appeared in recent literature or used in current practice. Even though we restrict to $L^{\infty}$, some examples, such as Value at Risk, can be defined on spaces of possibly unbounded random variables. Note that all acceptance sets below satisfy (2.2).

1. $\mathcal{A}=\left\{X \in L^{\infty} \mid X \geq m\right\}$, where $m \in \mathbb{R}$ is a given lower bound on the possible losses. Note that $\mathcal{A}$ is monotone and convex and it is the smallest acceptability set containing $m$. The corresponding simple capital requirement is then a convex risk measure and it is easily computed: $\rho_{\mathcal{A}}(X)=m-\operatorname{ess} . i n f X$. 
2. $\mathcal{A}=\mathcal{A}_{\alpha}=\left\{X \in L^{\infty} \mid P(X<0) \leq \alpha\right\}$ for a fixed $\alpha \in(0,1)$. The resulting simple capital requirement which is usually defined on the whole $L^{0}$ is the Value at Risk at level $\alpha$, denoted $\mathrm{VaR}_{\alpha}$, the most widespread risk measure in current practice. For any $X$, $\operatorname{VaR}_{\alpha}(X)$ coincides with the opposite of the upper quantile of order $\alpha$ of $X$. The set $\mathcal{A}$ is monotone and a positive cone, but it is not convex. Indeed, it is well known that $\mathrm{VaR}_{\alpha}$ is not a convex map.

3. $\mathcal{A}=\mathcal{A}_{\alpha}=\left\{X \in L^{\infty} \mid E_{P}\left(X I_{A}\right) \geq 0 \forall A \in \mathcal{F}\right.$ s.t. $\left.P(A)>\alpha\right\}$ for a fixed $\alpha \in(0,1)$. In this case $\mathcal{A}$ is a monotone and convex positive cone. The simple capital requirement is a coherent risk measure and coincides with the Worst Conditional Expectation as defined by Artzner et al. (1999), namely

$$
W C E_{\alpha}(X)=\sup \{-E(X \mid A) \mid A \in \mathcal{F}, P(A)>\alpha\} .
$$

4. $\mathcal{A}=\left\{X \in L^{\infty} \mid E_{P_{m}} X \geq 0\right.$ for $m=1, . ., M, E_{Q_{n}} X \geq F\left(Q_{n}\right)$, for $\left.n=1, . ., N\right\}$, where every $P_{m} \sim P$ is a valuation measure (for example an equivalent martingale measures in an incomplete market) and every $Q_{n} \ll P$ is a stress test measures with associated floors $F\left(Q_{n}\right)<0$. The set $\mathcal{A}$ is evidently monotone and convex; it is a positive cone provided all floors vanish. This example has been proposed in Carr et al. (2001).

5. If $\mathcal{A} \subset L^{\infty}$ is an acceptability set and $K$ is the convex cone of bounded attainable claims at zero cost in an incomplete market model, we define $\mathcal{A}_{K} \triangleq\left\{X \in L^{\infty} \mid X+f \in \mathcal{A}\right.$ for a $f \in K\}$. Then a payoff is acceptable for $\mathcal{A}_{K}$ if, trading on the market with a zero initial investment, we can make $X$ acceptable for $\mathcal{A}$. This example goes back to Föllmer and Schied (2002).

\subsection{On utility-based simple capital requirements}

Another important class of examples stems from the theory of expected utility. Let $u$ be a utility function satisfying:

$u: \mathbb{R} \rightarrow \mathbb{R}$ is concave, strictly increasing and $u(0)=0$.

For instance, we are considering linear $(u(x)=a x$ with $a>0)$ or exponential utility functions $\left(u(x)=1-e^{-\gamma x}\right.$ for a coefficient $\gamma>0$ describing risk aversion $)$. An agent whose preference structure is defined by the expected utility functional $U(X)=E_{P} u(X)$ can naturally define the following acceptability set:

$$
\mathcal{A}_{u} \triangleq\left\{X \in L^{\infty} \mid E_{P} u(X) \geq E_{P} u\left(X^{*}\right)\right\}
$$

where $X^{*}$ is a reference acceptable position. For formal simplification only we assume that $X^{*}=0$, so that $E_{P} u\left(X^{*}\right)=0$. Thanks to the hypothesis on $u$, the set $\mathcal{A}_{u}$ is monotone and convex. The associated capital requirement,

$$
\rho_{u}(X)=\inf \left\{\alpha \in \mathbb{R} \mid E_{P} u(X+\alpha) \geq 0\right\}
$$

is a finite valued map (see Föllmer and Schied 2002) and, of course, a convex risk measure. Also, the hypotheses on $u$ yield $\rho_{u}(0)=0$. When $u$ is linear, then $\rho_{u}(X)=-E_{P} X$, while in the 
case of exponential utility, $\rho_{u}$ is called an entropic risk measure. In this case we write $\rho_{u}=\rho_{\gamma}$, making the dependence on $\gamma$ explicit; a simple computation shows that

$$
\rho_{\gamma}(X)=\frac{1}{\gamma} \log E_{P} e^{-\gamma X}
$$

The following well-known dual representation holds:

$$
\rho_{\gamma}(X)=\sup _{Q \ll P}\left\{-E_{Q} X-\frac{1}{\gamma} H(Q \mid P)\right\}
$$

where $H(Q \mid P)$ is the relative entropy of $Q$ with respect to $P$. In the last section we will apply the following result which is shown in Proposition 3.10 by Barrieu and El Karoui (2005):

$$
\lim _{\gamma \rightarrow \infty} \rho_{\gamma}(X)=\sup _{Q \ll P: H(Q \mid P)<\infty}\left\{-E_{Q} X\right\}
$$

Basing on some experimental evidence, it has been argued that a measure of risk in general should not be considered, up to a sign, as an utility functional (see Brachinger and Weber 1997). In the proposition below we give a partial support to this statement showing that the preference structure defined by $E_{P} u(\cdot)$ does not coincide with the reverse ordering provided by $\rho_{u}$, unless $u$ is linear or exponential.

Proposition 2.8 Under the above assumptions on $u$, the following properties are equivalent:

1. for any $X, Y \in L^{\infty}: E_{P} u(X) \geq E_{P} u(Y)$ if and only if $\rho_{u}(X) \leq \rho_{u}(Y)$;

2. $u$ is linear or exponential.

Proof. $2 \Rightarrow 1$. We only have to check the validity of 1 . in the two cases. When $u$ is linear, this is trivial. When $u(x)=1-e^{-\gamma x}, \gamma>0$, then $E_{P} u(X) \geq E_{P} u(Y)$ if and only if $E_{P} e^{-\gamma X} \leq E_{P} e^{-\gamma Y}$. We then conclude thanks to (2.3).

$1 \Rightarrow 2$. If 1 . holds, then plainly there exists a strictly decreasing function $f: \mathbb{R} \rightarrow \mathbb{R}$ such that $E_{P} u(X)=f\left(\rho_{u}(X)\right)$ for any $X \in L^{\infty}$. In particular, for any $\alpha \in \mathbb{R}$ we have $u(\alpha)=E_{P} u(\alpha)=$ $f\left(\rho_{u}(\alpha)\right)=f(-\alpha)$, that is $f(\alpha)=u(-\alpha)$ and this means:

$$
E_{P} u(X)=u\left(-\rho_{u}(X)\right), \quad \forall X \in L^{\infty} .
$$

Since $u$ is strictly increasing, we can apply $u^{-1}$ on both sides of the previous equation, obtaining:

$$
C E_{u}(X) \triangleq u^{-1} E_{P} u(X)=-\rho_{u}(X), \quad \forall X \in L^{\infty},
$$

where $C E_{u}$ is the certainty equivalent. By translation invariance of $\rho_{u}$ we have

$$
C E_{u}(X+\alpha)=C E_{u}(X)+\alpha, \quad \forall X \in L^{\infty}, \alpha \in \mathbb{R} .
$$

We then conclude by applying the Kolmogorov-Nagumo-de Finetti theorem on associative means (see de Finetti, 1931) stating that (2.6) holds if and only if $u$ is linear or exponential. 


\section{General capital requirements}

\subsection{The definition}

We introduce in this section a general notion of capital requirement, generalizing the formulation (2.1) to a broader setting. Let $L$ be a vector space whose elements describe financial positions. For instance, $L$ can be a space of random variables, of deterministic functions, of stochastic processes and so on. We assume that $L$ is endowed with a partial vector order " $\leq$ ". Let $\mathcal{A} \subset L, \mathcal{C} \subset L$ and $\pi: \mathcal{C} \rightarrow \mathbb{R}$ be fixed.

Definition 3.1 The (general) capital requirement associated to $\mathcal{A}, \mathcal{C}$ and $\pi$ is the map $\rho_{\mathcal{A}, \mathcal{C}, \pi}$ : $L \rightarrow \mathbb{R}$ defined by

$$
\rho_{\mathcal{A}, \mathcal{C}, \pi}(x) \triangleq \inf \{\pi(y) \in \mathbb{R} \mid y \in \mathcal{C}, x+y \in \mathcal{A}\}, \quad x \in L,
$$

provided it is finite-valued.

In the next section we will provide some sufficient conditions for the finiteness of the map $\rho_{\mathcal{A}, \mathcal{C}, \pi}$, when $L$ is a space of bounded processes. For the time being, we assume that all capital requirements in this section are finite valued.

Clearly, when $L$ is a space of random variables, $\mathcal{C}=\mathbb{R}$ and $\pi=i d$, then $\rho_{\mathcal{A}, \mathcal{C}, \pi}$ coincides with the simple capital requirement $\rho_{\mathcal{A}}$. The economic interpretation of the quantity in (3.1) is as follows. The set $\mathcal{A}$ collects all acceptable positions, while $\mathcal{C}$ is the set of all positions which may be attained by means of permitted hedging strategies. Both $\mathcal{A}$ and $\mathcal{C}$ may be specified by an external regulator. The real amount $\pi(x)$ is the initial cost of the strategy leading to $x$; for instance it can be a market price of a claim or an amount which is invested in a saving account. The quantity $\rho_{\mathcal{A}, \mathcal{C}, \pi}(x)$ is then the minimal cost of a hedging strategy leading to $y \in \mathcal{C}$ which transform the original position $x \in L$ into an admissible one, i.e. $x+y \in \mathcal{A}$. In this section, we will not insist on the economic interpretation of (3.1), rather, we concentrate on some mathematical aspects, referring to Scandolo (2004) for some examples.

Remark 3.2 A natural generalization of the concept introduced in the above definition is given by:

$$
\rho_{\nu, \mathcal{C}, \pi}(x) \triangleq \inf \{\pi(y)+\nu(x+y) \mid y \in \mathcal{C}\}, \quad x \in L,
$$

where $\nu: L \rightarrow \mathbb{R} \cup\{+\infty\}$ is a convex functional that replaces in (3.1) the support functional $\delta_{\mathcal{A}}$ of the acceptance set $\mathcal{A}$. In this paper we will deal only with capital requirements in Definition (3.1), which are based on acceptance sets, and leave this generalization for further investigation.

\subsection{Properties}

Some natural properties of the characteristic sets $\mathcal{A}$ and $\mathcal{C}$ and of the mapping $\pi$ can be transferred to properties of the associated capital requirement.

Proposition 3.3 Let $\rho_{\mathcal{A}, \mathcal{C}, \pi}$ be the capital requirement associated to $\mathcal{A}, \mathcal{C}$ and $\pi$.

1. If $\pi$ is a convex map and $\mathcal{A}$ and $\mathcal{C}$ are convex sets, then $\rho_{\mathcal{A}, \mathcal{C}, \pi}$ is a convex map.

2. If $\mathcal{A}$ is a monotone set, i.e. $x \geq x^{\prime} \in \mathcal{A}$ implies $x \in \mathcal{A}$, then $\rho_{\mathcal{A}, \mathcal{C}, \pi}$ is monotone decreasing w.r.t. the partial order in $L$. 
3. If $\pi$ is positively homogeneous and $\mathcal{A}$ and $\mathcal{C}$ are positive cones, then $\rho_{\mathcal{A}, \mathcal{C}, \pi}$ is a positively homogeneous map.

Proof. 1. Let $x, x^{\prime} \in L$ and $\lambda \in[0,1]$. If $y, y^{\prime} \in \mathcal{C}$ are such that $x+y, x^{\prime}+y^{\prime} \in \mathcal{A}$, then convexity of $\mathcal{A}$ and $\mathcal{C}$ yields $\widehat{y} \triangleq \lambda y+(1-\lambda) y^{\prime} \in \mathcal{C}$ and $\widehat{y}+\lambda x+(1-\lambda) x^{\prime} \in \mathcal{A}$. Since $\pi$ is convex, we then have

$$
\begin{aligned}
& \lambda \rho_{\mathcal{A}, \mathcal{C}, \pi}(x)+(1-\lambda) \rho_{\mathcal{A}, \mathcal{C}, \pi}\left(x^{\prime}\right)= \\
& =\inf \left\{\lambda \pi(y)+(1-\lambda) \pi\left(y^{\prime}\right) \mid y, y^{\prime} \in \mathcal{C}, x+y, x^{\prime}+y^{\prime} \in \mathcal{A}\right\} \\
& \geq \inf \left\{\pi\left(\lambda y+(1-\lambda) y^{\prime}\right) \mid y, y^{\prime} \in \mathcal{C}, x+y, x^{\prime}+y^{\prime} \in \mathcal{A}\right\} \\
& \geq \inf \left\{\pi(\widehat{y}) \mid \widehat{y} \in \mathcal{C}, \widehat{y}+\lambda x+(1-\lambda) x^{\prime} \in \mathcal{A}\right\} \\
& =\rho_{\mathcal{A}, \mathcal{C}, \pi}\left(\lambda x+(1-\lambda) x^{\prime}\right),
\end{aligned}
$$

i.e. $\rho_{\mathcal{A}, \mathcal{C}, \pi}$ is a convex map.

2. Let $x, x^{\prime} \in L$ with $x \geq x^{\prime}$. Monotonicity of $\mathcal{A}$ yields the inclusion $\left\{y \in \mathcal{C} \mid x^{\prime}+y \in \mathcal{A}\right\} \subseteq\{y \in$ $\mathcal{C} \mid x+y \in \mathcal{A}\}$ and $\rho_{\mathcal{A}, \mathcal{C}, \pi}(x) \leq \rho_{\mathcal{A}, \mathcal{C}, \pi}\left(x^{\prime}\right)$ easily follows.

3. Let $x \in L$ and $\lambda>0$. Since $\mathcal{A}$ and $\mathcal{C}$ are positive cones, then $z \in \mathcal{A}$ if and only if $\lambda z \in \mathcal{A}$ and similarly for $\mathcal{C}$. Therefore:

$$
\begin{aligned}
\lambda \rho_{\mathcal{A}, \mathcal{C}, \pi}(x) & =\inf \{\lambda \pi(y)=\pi(\lambda y) \mid y \in \mathcal{C}, x+y \in \mathcal{A}\} \\
& =\inf \{\pi(\lambda y) \mid \lambda y \in \mathcal{C}, \lambda x+\lambda y \in \mathcal{A}\} \\
& =\inf \{\pi(\widehat{y}) \mid \widehat{y} \in \mathcal{C}, \lambda x+\widehat{y} \in \mathcal{A}\}=\rho_{\mathcal{A}, \mathcal{C}, \pi}(\lambda x)
\end{aligned}
$$

\subsection{The translation invariance property}

We begin with an abstract property inspired by translation invariance in the static setting.

Definition 3.4 A map $\rho: L \rightarrow \mathbb{R}$ is translation invariant with respect to $(\mathcal{W}, \pi)$, where $\mathcal{W} \subseteq L$ and $\pi: \mathcal{W} \rightarrow \mathbb{R}$ if:

$$
\rho(x+z)=\rho(x)-\pi(z) \quad \forall x \in L, z \in \mathcal{W} .
$$

When $L$ is a space of random variables, then the classical notion of translation invariance corresponds to the specification $\mathcal{W}=\mathbb{R}$ and $\pi=i d$. If the map $\rho$ has the meaning of a risk measure, the general property introduced above can be explained as follows. The set $\mathcal{W}$ collects a set of financial instruments and $\pi$ is their cost or price. Then (3.2) states that if a long position $z$ is added to the original position $x$, then the riskiness decreases exactly by the price of $z$. We will adopt the following notation: if $f: A \rightarrow \mathbb{R}$, then $f(A) \triangleq\{f(a) \mid a \in A\} \subseteq \mathbb{R}$.

Proposition 3.5 Let $\pi$ be linear. Any general capital requirement $\rho_{\mathcal{A}, \mathcal{C}, \pi}$ is translation invariant with respect to $(\mathcal{W}, \pi)$, where $\mathcal{W} \triangleq\{z \in \mathcal{C} \mid y \pm z \in \mathcal{C} \forall y \in \mathcal{C}\}$. 
Proof. First observe that if $z \in \mathcal{W}$, then $y \in \mathcal{C}$ if and only if $y-z \in \mathcal{C}$. Therefore, for any $x \in L$ it holds:

$$
\begin{aligned}
\rho_{\mathcal{A}, \mathcal{C}, \pi}(x+z) & =\inf \{\pi(y) \in \mathbb{R} \mid y \in \mathcal{C}, x+z+y \in \mathcal{A}\} \\
& =\inf \left\{\pi\left(y^{\prime}-z\right) \in \mathbb{R} \mid y^{\prime}-z \in \mathcal{C}, x+y^{\prime} \in \mathcal{A}\right\} \\
& =\inf \left\{\pi\left(y^{\prime}\right)-\pi(z) \in \mathbb{R} \mid y^{\prime} \in \mathcal{C}, x+y^{\prime} \in \mathcal{A}\right\} \\
& =\rho_{\mathcal{A}, \mathcal{C}, \pi}(x)-\pi(z) .
\end{aligned}
$$

We now generalize Proposition 2.3 and characterize those maps that admit a representation as capital requirements. If $\rho: L \rightarrow \mathbb{R}$ we set $\mathcal{A}_{\rho} \triangleq\{x \in L \mid \rho(x) \leq 0\}$.

Proposition 3.6 Let $\rho: L \rightarrow \mathbb{R}$ be translation invariant with respect to some $(\mathcal{W}, \pi)$ and assume that $\rho(L) \subseteq \pi(\mathcal{W})$. Then $\rho=\rho_{\mathcal{A}_{\rho}, \mathcal{W}, \pi}$.

Proof. We have, for any $x \in L$,

$$
\begin{aligned}
\rho_{\mathcal{A}_{\rho}, \mathcal{W}, \pi}(x) & =\inf \{\pi(y) \in \mathbb{R} \mid y \in \mathcal{W}, \rho(x+y) \leq 0\} \\
& =\inf \{\pi(y) \in \mathbb{R} \mid y \in \mathcal{W}, \rho(x) \leq \pi(y)\}=\rho(x) .
\end{aligned}
$$

\subsection{A dual representation result}

We address here the representability of a capital requirement in terms of linear functionals, which generalizes the corresponding representation of risk measures. Assume that $L$ is endowed with a locally convex topology $\tau$ compatible with a dual pair $\left\langle L, L^{\prime}\right\rangle$, where $L^{\prime}$ is a second vector space. If $\phi \in L^{\prime}$, then we will write $\phi(x)$ instead of $\langle x, \phi\rangle$.

Definition 3.7 A map $\rho: L \rightarrow \mathbb{R}$ is $\tau$-lower semicontinuous if the set $\{x \in L \mid \rho(x) \leq \alpha\}$ is $\tau$-closed for any $\alpha \in \mathbb{R}$.

Remark 3.8 Let $\rho: L \rightarrow \mathbb{R}$ be translation invariant with respect to some $(\mathcal{W}, \pi)$ and assume that $\rho(L) \subseteq \pi(\mathcal{W})$. From Proposition 3.6 it follows that $\rho$ is $\tau$-lower semicontinuous if $\mathcal{A}_{\rho}$ is $\tau$-closed.

We already stated in Proposition 3.3 the sufficient conditions under which a capital requirement is a convex map.

Proposition 3.9 Let $\mathcal{A}, \mathcal{C}$ and $\pi$ be fixed. If $\rho_{\mathcal{A}, \mathcal{C}, \pi}$ is convex and $\tau$-lower semicontinuous, then, for any $x \in L$

$$
\rho_{\mathcal{A}, \mathcal{C}, \pi}(x)=\sup _{\phi \in \mathcal{D}}\left\{-\phi(x)-\gamma_{\mathcal{A}}(\phi)-\gamma_{\mathcal{C}, \pi}(\phi)\right\},
$$

where

$$
\begin{aligned}
\gamma_{\mathcal{A}}(\phi) & \triangleq \sup _{x \in \mathcal{A}}\{-\phi(x)\} \\
\gamma_{\mathcal{C}, \pi}(\phi) & \triangleq \sup _{y \in \mathcal{C}}\{\phi(y)-\pi(y)\} \\
\mathcal{D} & \triangleq \operatorname{dom} \gamma_{\mathcal{A}} \cap \operatorname{dom} \gamma_{\mathcal{C}, \pi} \triangleq \mathcal{D}_{\mathcal{A}} \cap \mathcal{D}_{\mathcal{C}, \pi}
\end{aligned}
$$


Proof. The map $\rho_{\mathcal{A}, \mathcal{C}, \pi}$ is convex and lower semicontinuous with respect to a topology compatible with $\left\langle L, L^{\prime}\right\rangle$, hence the Fenchel-Moreau Theorem yields

$$
\rho_{\mathcal{A}, \mathcal{C}, \pi}(x)=\sup _{\phi \in L^{\prime}}\left\{-\phi(x)-\rho_{\mathcal{A}, \mathcal{C}, \pi}^{*}(-\phi)\right\}
$$

where $\rho_{\mathcal{A}, \mathcal{C}, \pi}^{*}$ is the convex-conjugated of $\rho_{\mathcal{A}, \mathcal{C}, \pi}$. Moreover we have:

$$
\begin{aligned}
\rho_{\mathcal{A}, \mathcal{C}, \pi}^{*}(-\phi) & \triangleq \sup _{x \in L}\left\{-\phi(x)-\rho_{\mathcal{A}, \mathcal{C}, \pi}(x)\right\}= \\
& =\sup _{x \in L}\{-\phi(x)-\inf \{\pi(y) \in \mathbb{R} \mid y \in \mathcal{C}, x+y \in \mathcal{A}\}\}= \\
& =\sup \{-\phi(x)-\pi(y) \mid x \in L, y \in \mathcal{C}, x+y \in \mathcal{A}\}= \\
& =\sup \{-\phi(z-y)-\pi(y) \mid y \in \mathcal{C}, z \in \mathcal{A}\}= \\
& =\sup \{-\phi(z)+\phi(y)-\pi(y) \mid z \in \mathcal{A}, y \in \mathcal{C}\}= \\
& =\sup _{x \in \mathcal{A}}\{-\phi(x)\}+\sup _{y \in \mathcal{C}}\{\phi(y)-\pi(y)\} .
\end{aligned}
$$

Finally, observe that only those functionals in $\mathcal{D}$ do enter the representation.

We see that the acceptance set $\mathcal{A}$ enters in the above representation only through its associated penalty function $\gamma_{\mathcal{A}}(\phi)$, while the set $\mathcal{C}$ of positions from permitted hedging strategies and the cost functional $\pi$ determine the second penalty function $\gamma_{\mathcal{C}, \pi}(\phi)$.

If $\mathcal{B} \subseteq L$ is a convex subset, then its recession cone $0^{+} \mathcal{B}$ is defined by (see Rockafellar, 1970):

$$
0^{+} \mathcal{B} \triangleq\{v \in L \mid x+\lambda v \in \mathcal{B}, \forall x \in \mathcal{B}, \lambda \geq 0\}
$$

Note that $0^{+} \mathcal{B}$ is a (positive) convex cone containing 0 and that $0^{+} \mathcal{B} \subseteq \mathcal{B}$ provided $0 \in \mathcal{B}$. It indicates the possible directions in which $\mathcal{B}$ is "unbounded"; therefore, it may well reduce to $\{0\}$. A map $\psi: \mathcal{B} \rightarrow \mathbb{R}$ is super-linear if $\psi(x+y) \geq \psi(x)+\psi(y)$ for any $x, y \in \mathcal{B}$ such that $x+y \in \mathcal{B}$ and if $\psi(\lambda x) \geq \lambda \psi(x)$ for any $\lambda \geq 0$ and $x \in \mathcal{B}$ such that $\lambda x \in \mathcal{B}$. Changing the inequalities we can define sub-linear maps. In particular, any linear form is both super-linear and sub-linear.

Lemma 3.10 (i) Let $\psi$ be super-linear on $\mathcal{B}$. If $\sup _{x \in \mathcal{B}} \psi(x)<+\infty$, then

$$
\psi(y) \leq 0 \quad \forall y \in 0^{+} \mathcal{B} .
$$

(ii) If $\mathcal{A}$ is monotone, then $\mathcal{D}_{\mathcal{A}} \subseteq L_{+}^{\prime} \triangleq\left\{\phi \in L^{\prime} \mid \phi(x) \geq 0 \forall x \in L_{+}\right\}$.

(iii) If $\pi$ is sub-linear, then $\mathcal{D}_{\mathcal{C}, \pi} \subseteq\left\{\phi \in L^{\prime} \mid \phi(y) \leq \pi(y) \forall y \in 0^{+} \mathcal{C}\right\}$.

Proof. (i) Assume that $\psi(y)>0$ for some $y \in 0^{+} \mathcal{B}$. Then

$$
\sup _{x \in \mathcal{B}} \psi(x) \geq \sup _{\lambda \geq 0} \psi(x+\lambda y) \geq \psi(x)+\sup _{\lambda \geq 0} \psi(\lambda y) \geq \psi(x)+\sup _{\lambda \geq 0} \lambda \psi(y)=+\infty .
$$

(ii) It is easy to prove that $\mathcal{A}$ is monotone, i.e. $x \geq y \in \mathcal{A} \Rightarrow x \in \mathcal{A}$, if and only if $L_{+} \subseteq 0^{+} \mathcal{A}$. If $\phi \in \mathcal{D}_{\mathcal{A}}$, then by (i), it follows $-\phi(y) \leq 0$ for any $y \in L_{+} \subseteq 0^{+} \mathcal{A}$. (iii) The map $\psi=\phi-\pi$ satisfies the hypothesis in (i). It then follows $\psi(y) \leq 0$ for any $y \in 0^{+} \mathcal{C}$ and we conclude.

The next proposition will be useful in the following sections. 
Proposition 3.11 Let $\pi$ be sub-linear, $\mathcal{C}$ convex, $\mathcal{A}$ convex and monotone. If the convex map $\rho_{\mathcal{A}, \mathcal{C}, \pi}$ is $\tau$-lower semicontinuous, then (3.3) holds true and

$$
\mathcal{D} \subseteq\left\{\phi \in L_{+}^{\prime} \mid \phi(y) \leq \pi(y) \forall y \in 0^{+} \mathcal{C}\right\} .
$$

If in addition $\mathcal{C}$ is a positive cone and $\pi(0)=0$, then $\gamma_{\mathcal{C}}$ vanishes on $\mathcal{D}$.

Proof. We have just to put together Propositions 3.3 and 3.9, Lemma 3.10 (ii) and (iii) and observe that every sub-linear map is convex. If $\mathcal{C}$ is a positive cone, then $0^{+} \mathcal{C}=\mathcal{C}$. Henceforth $\gamma_{\mathcal{C}, \pi} \leq 0$ on $\mathcal{D}$ just by definition. However, the equality is actually attained as $0 \in 0^{+} \mathcal{C}=\mathcal{C}$ and $\phi(0)-\pi(0)=0$ for any $\phi \in L^{\prime}$.

\section{The multi-period setting}

We now turn our attention to a multi-period setting, in which the financial positions to be considered have also a temporal dimension being them described by stochastic processes instead of simple random variables.

\subsection{Set-up}

A set of subsequent dates $\left(t_{n}\right)_{n=1}^{N}$ is fixed, with $0=t_{0}<t_{n}<t_{n+1}$ for any $n \geq 1$ and $t_{N}=T$. We therefore add to the probability space $(\Omega, \mathcal{F}, P)$, a filtration $\mathbb{F}=\left(\mathcal{F}_{n}\right)_{n=0}^{N}$, where $\mathcal{F}_{n}$ describes the information available at date $t_{n}$. Naturally, we will assume that $\mathcal{F}_{0}$ is trivial and that $\mathcal{F}_{N}=\mathcal{F}$; if $X$ is a random variable, we will write $X \in \mathbb{R}$ to say that $X$ is $\mathcal{F}_{0}$-measurable. The set

$$
\mathcal{P}_{n} \triangleq\left\{Q \text { is a probability on }\left(\Omega, \mathcal{F}_{n}\right) \text { such that } Q \ll P\right\}
$$

collects all probabilistic models for the uncertainty to date $t_{n}$. Let $\mathcal{L}^{0}=\mathcal{L}^{0}(\Omega, \mathcal{F}, P, \mathbb{F})$ be the space of (discrete-time) adapted processes starting in $t_{1}$, i.e.

$$
\mathcal{L}^{0} \triangleq\left\{\mathbf{X}=\left(X_{1}, \ldots, X_{N}\right) \mid X_{n} \in L^{0}\left(\Omega, \mathcal{F}_{n}, P\right) \forall n\right\}
$$

and, for any $p \in[1,+\infty]$, define

$$
\mathcal{L}^{p} \triangleq\left\{\mathbf{X} \in \mathcal{L}^{0} \mid X_{n} \in L^{p}\left(\Omega, \mathcal{F}_{n}, P\right) \forall n\right\}
$$

We set $\mathbf{0}=(0, \ldots, 0), \mathbf{1}=(1, \ldots, 1)$ and, for any fixed $n$ between 1 and $N, \mathbf{e}^{n}=(0, \ldots, 1, \ldots, 0)$, where 1 occupies the $n$th position. We will write $\mathbf{X} \geq \mathbf{Y}$ for $X_{n} \geq Y_{n} \forall n$.

\subsection{Risk measures for processes}

We can interpret a process in $\mathcal{L}^{0}$ in three different ways:

- $\mathbf{X}$ is a payoff stream: $X_{n}$ is the net payoff liquidated to an agent at time $t_{n}$. As in the one-period setting, positive as well as negative values are allowed and positive values correspond to gains.

- $\widehat{\mathbf{X}}$ is the evolution of a cumulated payoff: $\widehat{X}_{n}=\sum_{i=1}^{n} X_{i}$, where $\mathbf{X}$ is a payoff stream. 
- $\widetilde{\mathbf{X}}$ is the evolution of a portfolio value: $\widetilde{X}_{n}=V_{n}$, where $V_{n}$ is the market (or book, or liquidation) value of a portfolio at time $t_{n}$. Also, $\widetilde{X}_{n}$ could be considered as the relative change: $\widetilde{X}_{n}=V_{n}-V_{0}$.

Whatever the interpretation of the processes are, we can define a risk measure on $\mathcal{L}^{\infty}$ as any map $\rho: \mathcal{L}^{\infty} \rightarrow \mathbb{R}$ that assigns to each position $\mathbf{X} \in \mathcal{L}^{\infty}$ its riskiness $\rho(\mathbf{X})$. Three out of the four coherency axioms listed in Section 2 can be immediately generalized to the multi-period setting. They are:

1. Monotonicity: $\mathbf{X} \geq \mathbf{Y}$ implies $\rho(\mathbf{X}) \leq \rho(\mathbf{Y})$

2. Convexity: $\rho(\lambda \mathbf{X}+(1-\lambda) \mathbf{Y}) \leq \lambda \rho(\mathbf{X})+(1-\lambda) \rho(\mathbf{Y})$ for any $\mathbf{X}, \mathbf{Y} \in \mathcal{L}^{\infty}$ and $\lambda \in[0,1]$.

3. Positive homogeneity: $\rho(\lambda \mathbf{X})=\lambda \rho(\mathbf{X})$, for any $\mathbf{X} \in \mathcal{L}^{\infty}$ and $\lambda \geq 0$.

The economic motivation of the above three properties is the same as in the one-period case and, similarly, criticism may apply to homogeneity. Even though these axioms are reasonable under any of the financial interpretation of the processes, we stress that from now on we adopt only the interpretation of $X$ as a payoff stream.

Remark 4.1 There is clearly a one-to-one correspondence between risk measures for payoff streams and for cumulated sums. Indeed, if $\rho$ acts on payoff streams, then $\rho^{c}(\widehat{\mathbf{X}}) \triangleq \rho\left(\widehat{X}_{1}, \widehat{X}_{2}-\right.$ $\left.\widehat{X}_{1}, \ldots, \widehat{X}_{N}-\widehat{X}_{N-1}\right)$ acts on cumulated sums; conversely, if $\rho^{c}$ acts on cumulated sums, then $\rho(\mathbf{X}) \triangleq \rho^{c}\left(X_{1}, X_{1}+X_{2}, \ldots, \sum_{n=1}^{N} X_{n}\right)$ acts on payoff streams. This correspondence preserves convexity and homogeneity. Note that if $\rho^{c}$ is monotone $\left(\widehat{\mathbf{X}} \geq \widehat{\mathbf{Y}}\right.$ implies $\left.\rho^{c}(\widehat{\mathbf{X}}) \leq \rho^{c}(\widehat{\mathbf{Y}})\right)$ then also $\rho(\mathbf{X}) \triangleq \rho^{c}(\widehat{\mathbf{X}})$ is monotone. However, the converse is in general not true. Hence, monotonicity of $\rho$ (defined on payoff streams) is weaker than monotonicity of $\rho^{c}$ (defined on cumulated payoffs.)

Remark 4.2 The definitions of translation invariance and capital requirement we will give below are tailor-made for the case of payoff streams. Artzner et al. (2004) discuss the interpretation of $\mathbf{X}$ as a portfolio value, proposing a different and formally simpler notion of translation invariance and capital requirement (see also Remark 4.9).

\subsection{A class of capital requirements for processes}

We propose here a class of capital requirements for processes, specializing the definition (CR) and assuming that the agent has no access to an underlying market, but only to a saving account. We choose to call them standard capital requirements, since they correspond to the simplest financial framework, as we will see. In the present setting and within definition (3.1), this class corresponds to the specifications:

$$
\begin{aligned}
& \mathcal{C} \subseteq\left\{\mathbf{Y} \in \mathcal{L}^{\infty} \mid \sum_{n=1}^{N} Y_{n} \in \mathbb{R}\right\}, \\
& \pi(\mathbf{Y}) \triangleq \sum_{n=1}^{N} Y_{n} \in \mathbb{R}, \text { for } \mathbf{Y} \in \mathcal{L}^{\infty} .
\end{aligned}
$$


Denoting

$$
\mathcal{L}_{\Sigma}^{\infty} \triangleq\left\{\mathbf{X} \in \mathcal{L}^{\infty} \mid \sum_{n=1}^{N} X_{n} \in \mathbb{R}\right\}
$$

we thus consider maps $\rho_{\mathcal{A}, \mathcal{C}}: \mathcal{L}^{\infty} \rightarrow \mathbb{R}$ of the form

$$
\rho_{\mathcal{A}, \mathcal{C}}(\mathbf{X}) \triangleq \inf \left\{\sum_{n=1}^{N} Y_{n} \in \mathbb{R} \mid \mathbf{Y} \in \mathcal{C} \subseteq \mathcal{L}_{\Sigma}^{\infty}, \mathbf{X}+\mathbf{Y} \in \mathcal{A}\right\} .
$$

Definition 4.3 The standard capital requirement associated to $\mathcal{A} \subseteq \mathcal{L}^{\infty}$ and $\mathcal{C} \subseteq \mathcal{L}_{\Sigma}^{\infty}$ is the map $\rho_{\mathcal{A}, \mathcal{C}}$ defined by (4.2), provided it is finite valued (see below). When $\mathcal{C}=\mathcal{L}_{\Sigma}^{\infty}$ we will simply write $\rho_{\mathcal{A}}$.

Observe that when $N=1$, then these capital requirements coincide with those of Definition 2.2 .

The acceptability set of processes $\mathcal{A} \subset \mathcal{L}^{\infty}$ that collects all payoff streams which are deemed sustainable is fixed, maybe by an external regulator. We will provide some concrete examples in Section 5; for the time being we make a natural monotonicity assumption on $\mathcal{A}$, which has the same economic rationale as in the one-period setting.

Assumption $\mathbf{A}$. The set $\mathcal{A}$ is monotone, i.e. $\mathbf{X} \geq \mathbf{Y} \in \mathcal{A}$ implies $\mathbf{X} \in \mathcal{A}$.

\section{Operative meaning}

In order to explain the operative meaning of the Definition 4.3, we begin with the capital requirements $\rho_{\mathcal{A}}$. At time $t_{0}$, an agent who faces a payoff stream $\mathbf{X} \in \mathcal{L}^{\infty}$ can invest in a saving account the sum $V_{0} \in \mathbb{R}$ and then plan a disinvestment strategy $\mathbf{Y} \in \mathcal{L}^{\infty}$. When $Y_{n}$ is positive, then it represents the amount which will be disinvested from the bank account at time $t_{n}$. When $Y_{n}$ is negative, then $-Y_{n}>0$ is the amount which will be invested at time $t_{n}$. We stress that $Y_{n}$ is in principle a random quantity, meaning that the agent decides its amount according to future information (up to time $t_{n}$ ). In any case, the process $\mathbf{Y}$ is an additional payoff stream and the agent seeks for those $\mathbf{Y}$ which make the overall stream acceptable, i.e.:

$$
\mathbf{X}+\mathbf{Y}=\left(X_{1}+Y_{1}, \ldots, X_{N}+Y_{N}\right) \in \mathcal{A} .
$$

Starting with an initial investment $V_{0}$ and adopting the strategy $\mathbf{Y} \in \mathcal{L}^{\infty}$, the value of the account in $t_{n}, n \geq 1$, is given by:

$$
V_{n}\left(\mathbf{Y}, V_{0}\right)=V_{n-1}\left(\mathbf{Y}, V_{0}\right)-Y_{n}=V_{0}-\sum_{i=1}^{n} Y_{i}
$$

In principle, the account may take negative values at any date, in particular $V_{0}$ may be negative. This means that the agent is allowed to take out a loan. However, since the loan has to be repaid back before the last date, the account value cannot be negative at the last date, i.e.

$$
V_{N}\left(\mathbf{Y}, V_{0}\right)=V_{0}-\sum_{n=1}^{N} Y_{n} \geq 0 .
$$

An agent is clearly interested in the minimal initial endowment $V_{0}$ that admits a disinvestment strategy $\mathbf{Y} \in \mathcal{L}^{\infty}$ satisfying (4.3) and (4.5), i.e. in the quantity:

$$
\widehat{\rho}_{\mathcal{A}}(\mathbf{X}) \triangleq \inf \left\{V_{0} \in \mathbb{R} \mid \exists \mathbf{Y} \in \mathcal{L}^{\infty} \text { s.t. } V_{N}\left(\mathbf{Y}, V_{0}\right) \geq 0, \mathbf{X}+\mathbf{Y} \in \mathcal{A}\right\} .
$$


Proposition 4.4 Under Assumption A, the maps $\widehat{\rho}_{\mathcal{A}}$ and $\rho_{\mathcal{A}}$ coincide.

Proof. As a first step, note that

$$
\rho_{\mathcal{A}}(\mathbf{X})=\inf \left\{V_{0} \in \mathbb{R} \mid \exists \mathbf{Y} \in \mathcal{L}^{\infty} \text { s.t. } V_{N}\left(\mathbf{Y}, V_{0}\right)=0, \mathbf{X}+\mathbf{Y} \in \mathcal{A}\right\}
$$

Then the inequality $\widehat{\rho}_{\mathcal{A}} \leq \rho_{\mathcal{A}}$ is trivial. Conversely, fix $V_{0} \in \mathbb{R}$ and assume that $\mathbf{Y} \in \mathcal{L}^{\infty}$ satisfies (4.3) and (4.5). Define $\mathbf{Y}^{\prime} \in \mathcal{L}^{\infty}$ as $Y_{n}^{\prime} \triangleq Y_{n}$ for any $n \leq N-1$ and $Y_{N}^{\prime} \triangleq Y_{N}+V_{N}\left(\mathbf{Y}, V_{0}\right) \geq Y_{N}$, so that $V_{N}\left(\mathbf{Y}^{\prime}, V_{0}\right)=0$. Since $\mathbf{Y}^{\prime} \geq \mathbf{Y}$, by monotonicity of $\mathcal{A}$ we have $\mathbf{X}+\mathbf{Y}^{\prime} \in \mathcal{A}$, so that $\mathbf{Y}^{\prime}$ too satisfies (4.3) and (4.5) and the inequality " $\geq$ " is proved.

The subspace $\mathcal{L}_{\Sigma}^{\infty}$ collects all strategies that provide a complete disinvestment of the initial endowment $V_{0}=\sum_{n=1}^{N} Y_{n}$ and repay back every debt. However, within the same operative scheme, a regulation could restrict the set of permitted disinvestment strategies to $\mathcal{C} \subset \mathcal{L}_{\Sigma}^{\infty}$, leading the agent to consider more general standard capital requirements $\rho_{\mathcal{A}, \mathcal{C}}$ as defined in (4.2).

\section{The finiteness of the map $\rho_{\mathcal{A}, \mathcal{C}}$}

Consider two other natural assumptions, on $\mathcal{A}$ and $\mathcal{C}$, respectively.

Assumption B. There exists $\gamma \in \mathbb{R}$ such that $\sum_{n=1}^{N} X_{n} \leq \gamma$ implies $\mathbf{X} \notin \mathcal{A}$.

This assumption says that a payoff stream whose cumulated value is too low is not acceptable and therefore it seems highly reasonable. We stress that, on the contrary, the "symmetric" property (a payoff process with high enough cumulated value is acceptable) is much less reasonable from a financial point of view. The second assumption concerns the set of permitted strategies.

Assumption C. The set $\mathcal{C}$ contains $\mathcal{C}_{\mathbb{R}_{+}} \triangleq\left\{\mathbf{Y} \in \mathcal{L}_{\Sigma}^{\infty} \mid Y_{n} \in \mathbb{R}, Y_{n} \geq 0 \forall n\right\}$.

The set $\mathcal{C}_{\mathbb{R}_{+}}$collects all deterministic strategies which involve only pure disinvestments, in particular $\mathbf{0}$, the "do-nothing" strategy. In particular, this means that $V_{n}\left(\mathbf{Y}, V_{0}\right) \geq 0, \mathbf{Y} \in \mathcal{C}_{\mathbb{R}_{+}}$, as it is easy to see. Since these strategies are the simplest ones and do not involve any exposure versus any counterpart, it is natural to include them in any specification of $\mathcal{C}$.

Proposition 4.5 Under Assumptions $A$ and $B$ (resp. $A$ and $C$ ) it holds $\rho_{\mathcal{A}, \mathcal{C}}(\mathbf{X})>-\infty$ (resp. $<+\infty$ ) for any $\mathbf{X} \in \mathcal{L}^{\infty}$.

Proof. Let A and B hold with constant $\gamma \in \mathbb{R}$. For any given $\mathbf{X} \in \mathcal{L}^{\infty}$, we claim that

$$
\rho_{\mathcal{A}, \mathcal{C}}(\mathbf{X}) \geq \alpha \triangleq \gamma-\sum_{n=1}^{N}\left\|X_{n}\right\|_{\infty}>-\infty .
$$

Indeed, if $\mathbf{Y} \in \mathcal{C}$ is such that $\sum_{n=1}^{N} Y_{n}<\alpha$, then $\sum_{n=1}^{N}\left(X_{n}+Y_{n}\right) \leq \sum_{n=1}^{N}\left(\left\|X_{n}\right\|_{\infty}+\alpha\right)=\gamma$. By $\mathrm{A}$ it follows that $\mathbf{X}+\mathbf{Y} \notin \mathcal{A}$ and (4.7) is proved.

Let now $\mathrm{A}$ and $\mathrm{C}$ hold. Fix $\mathbf{Z} \in \mathcal{A}$ and let us prove that for any $\mathbf{X} \in \mathcal{L}^{\infty}$ :

$$
\rho_{\mathcal{A}, \mathcal{C}}(\mathbf{X}) \leq \sum_{n=1}^{N}\left(\left\|X_{n}\right\|_{\infty}+\left\|Z_{n}\right\|_{\infty}\right)<+\infty .
$$


Indeed, define $Y_{n} \triangleq\left\|X_{n}\right\|_{\infty}+\left\|Z_{n}\right\|_{\infty} \geq 0$ for any $n$, observing that $X_{n}+Y_{n} \geq\left\|Z_{n}\right\|_{\infty} \geq Z_{n}$. Then $\mathbf{Y} \in \mathcal{C}_{\mathbb{R}_{+}} \subseteq \mathcal{C}$ by $\mathrm{C}$ and $\mathbf{X}+\mathbf{Y} \geq \mathbf{Z} \in \mathcal{A}$ by A. Finally, $\sum_{n=1}^{N} Y_{n}=\sum_{n=1}^{N}\left(\left\|X_{n}\right\|_{\infty}+\left\|Z_{n}\right\|_{\infty}\right)$, hence (4.8).

\section{Additional properties}

Consider the following additional assumptions on $\mathcal{A}$ and $\mathcal{C}$ :

Assumption D. The sets $\mathcal{A}$ and $\mathcal{C}$ are convex.

Assumption E. The sets $\mathcal{A}$ and $\mathcal{C}$ are positive cones.

Assumption D seems to be reasonable: the convex combination of two acceptable streams or permitted strategies should be acceptable or permitted as well. On the contrary, Assumption E is debatable, since in some cases multiplying an acceptable stream by a large (positive) constant may introduce liquidity risk. We will see below an important example in which $\mathcal{C}$ is not a positive cone.

Proposition 4.6 Under Assumption A (resp. D, resp. E) $\rho_{\mathcal{A}, \mathcal{C}}$ is monotone (resp. convex, resp. positively homogeneous).

Proof. Since the map $\pi$ is linear on $\mathcal{C}$, these are immediate consequences of Proposition 3.3.

\subsection{Translation invariance}

Here, we are specializing to the present setting the general notion of translation invariance introduced in the previous section.

Definition 4.7 A map $\rho: \mathcal{L}^{\infty} \rightarrow \mathbb{R}$ is translation invariant with respect to a given $\mathcal{W} \subseteq \mathcal{L}_{\Sigma}^{\infty}$, or simply $\mathcal{W}$-invariant, if

$$
\rho(\mathbf{X}+\mathbf{Z})=\rho(\mathbf{X})-\sum_{n=1}^{N} Z_{n} \quad \forall \mathbf{X} \in \mathcal{L}^{\infty}, \mathbf{Z} \in \mathcal{W} .
$$

Remark 4.8 This property clearly coincide with translation invariance with respect to $(\mathcal{W}, \pi)$ as in Definition 3.4, when $\pi(\mathbf{Z})=\sum_{n=1}^{N} Z_{n}$. If $N=1$ and $\mathcal{W}=\mathcal{L}_{\Sigma}^{\infty} \simeq \mathbb{R}$, then we recover the classical definition of translation invariance.

Remark 4.9 Riedel (2002) and Weber (2004) propose, as a part of the definition of a dynamic risk measure, a common definition of translation invariance for the risk measurement at time $t_{0}$. In the present terminology, they require that a risk measure is $\mathcal{W}$-invariant for the set $\mathcal{W}=\mathcal{C}_{\mathbb{R}_{+}}$ defined in the Assumption $\mathrm{C}$ above.

Proposition 4.10 Let $\mathcal{W} \subseteq \mathcal{L}_{\Sigma}^{\infty}$ be a subspace containing $\mathcal{C}_{\mathbb{R}_{+}}$. Then a risk measure $\rho$ is $\mathcal{W}$ invariant if and only if $\rho \equiv \rho_{\mathcal{A}, \mathcal{W}}$ for some $\mathcal{A} \subseteq \mathcal{L}^{\infty}$. 
Proof. Let $\mathcal{A} \subset \mathcal{L}^{\infty}$. Since $\mathcal{W}$ is a subspace, then $\mathcal{W}=\mathcal{W}^{\prime} \triangleq\{Z \in \mathcal{W} \mid Y \pm Z \in \mathcal{W} \forall Y \in \mathcal{W}\}$. Moreover, $\pi$ is linear so that we can apply Proposition 3.5, deducing that $\rho=\rho_{\mathcal{A}, \mathcal{W}}$ is $\mathcal{W}$ invariant. Conversely, let $\rho$ be $\mathcal{W}$-invariant. Since $\mathcal{W}$ is a subspace and contains $\mathcal{C}_{\mathbb{R}_{+}}$, then $\lambda \mathbf{e}^{1} \in \mathcal{W}$ for any $\lambda \in \mathbb{R}$, so that $\pi(\mathcal{W}) \subseteq\left\{\lambda=\pi\left(\lambda \mathbf{e}^{1}\right) \mid \lambda \in \mathbb{R}\right\}=\mathbb{R}$. Hence the conditions of Proposition 3.6 are surely met and we conclude that $\rho=\rho_{\mathcal{A}, \mathcal{W}}$ for some $\mathcal{A}$.

\subsection{The dual representation}

For any pair $p, q \in[1,+\infty]$ of conjugated exponents the non-degenerate bilinear form

$$
\langle\mathbf{X}, \mathbf{Z}\rangle \triangleq \sum_{n=1}^{N} E_{P}\left(X_{n} Z_{n}\right), \quad \mathbf{X} \in \mathcal{L}^{p}, \mathbf{Z} \in \mathcal{L}^{q} .
$$

puts $\mathcal{L}^{p}$ and $\mathcal{L}^{q}$ in natural duality. The following Lemma will be useful below; we omit the standard proof.

Lemma 4.11 In the duality $\left\langle\mathcal{L}^{\infty}, \mathcal{L}^{1}\right\rangle$, a linear form $\phi$ is non-negative if and only if it can be written as:

$$
\phi(\mathbf{X})=\phi_{\mathbf{c}, \mathbf{Q}}(\mathbf{X}) \triangleq \sum_{n=1}^{N} c_{n} E_{Q_{n}} X_{n}
$$

where $(\mathbf{c}, \mathbf{Q}) \in \mathcal{B} \triangleq\left\{\left(c_{n}, Q_{n}\right)_{n=1}^{N} \mid c_{n} \in \mathbb{R}_{+}, Q_{n} \in \mathcal{P}_{n} \forall n\right\}$.

Let $\tau$ be a topology compatible with the duality $\left\langle\mathcal{L}^{\infty}, \mathcal{L}^{1}\right\rangle$. Remind that $0^{+} \mathcal{C}$ denotes the recession cone of $\mathcal{C}$ as defined in (3.7).

Proposition 4.12 Under the Assumptions $A$ to $D$ and if $\rho_{\mathcal{A}, \mathcal{C}}$ is $\tau$-lower semicontinuous then

$$
\rho_{\mathcal{A}, \mathcal{C}}(\mathbf{X})=\sup _{(\mathbf{c}, \mathbf{Q}) \in \mathcal{B}_{\mathcal{C}}}\left\{-\sum_{n=1}^{N} c_{n} E_{Q_{n}} X_{n}-\gamma_{\mathcal{A}}(\mathbf{c}, \mathbf{Q})-\gamma_{\mathcal{C}}(\mathbf{c}, \mathbf{Q})\right\}, \quad \forall \mathbf{X} \in \mathcal{L}^{\infty},
$$

where

$$
\begin{aligned}
& \gamma_{\mathcal{A}}(\mathbf{c}, \mathbf{Q}) \triangleq \sup _{\mathbf{Y} \in \mathcal{A}}\left\{-\sum_{n=1}^{N} c_{n} E_{Q_{n}} Y_{n}\right\}, \\
& \gamma_{\mathcal{C}}(\mathbf{c}, \mathbf{Q}) \triangleq \sup _{\mathbf{Y} \in \mathcal{C}}\left\{\sum_{n=1}^{N}\left(c_{n} E_{Q_{n}} Y_{n}-Y_{n}\right)\right\}, \\
& \mathcal{B}_{\mathcal{C}} \triangleq\left\{(\mathbf{c}, \mathbf{Q}) \in \mathcal{B} \mid \sum_{n=1}^{N}\left(c_{n} E_{Q_{n}} Y_{n}-Y_{n}\right) \leq 0, \forall \mathbf{Y} \in 0^{+} \mathcal{C}\right\} .
\end{aligned}
$$

Proof. By Assumptions A to D, we know that $\rho_{\mathcal{A}, \mathcal{C}}$ is finite-valued, convex and monotone. Representation (4.12) is then a consequence of Proposition 3.11 and of Lemma 4.11 above.

Remark 4.13 Representation results similar to (4.12), in which the penalty function splits into two parts, have been derived by Föllmer and Schied (2002) for a convex risk measure in a one-period model, when an underlying financial market is available, and by Staum (2004) who considers bid/ask prices in an abstract setting. 


\section{$5 \quad$ A measurability constraint}

In this section we restrict the set of permitted disinvestment strategies by introducing a measurability constraint. Sometime, a regulator wants the disinvestment strategy of the agent to be planned at the initial date $t_{0}$ or, at least, by a specified date $t_{M}$, with $0 \leq M \leq N$. Therefore, we now consider the following subset of permitted strategies:

$$
\mathcal{C}=\mathcal{C}_{M} \triangleq\left\{\mathbf{Y} \in \mathcal{L}_{\Sigma}^{\infty} \mid Y_{n} \text { is } \mathcal{F}_{M} \text {-measurable } \forall n \geq M\right\} .
$$

Note that $\mathcal{C}_{N-1}=\mathcal{C}_{N}=\mathcal{L}_{\Sigma}^{\infty}$ and that, for any $M, \mathcal{C}_{M}$ is a subspace and therefore $0^{+} \mathcal{C}_{M}=$ $\mathcal{C}_{M}$. Let $\mathcal{A} \subset \mathcal{L}^{\infty}$ and set $\rho_{\mathcal{A}, M} \triangleq \rho_{\mathcal{A}, \mathcal{C}_{M}}$, as defined in (4.2) above. We specialize the dual representation in (4.12) to this class of capital requirements.

Proposition 5.1 Let $0 \leq M \leq N-2$. Assume that $\mathcal{A}$ is convex, monotone and satisfies assumption $B$ and that $\rho_{\mathcal{A}, M}$ is lower semicontinuous. Then it holds:

$$
\rho_{\mathcal{A}, M}(\mathbf{X})=\sup _{\mathbf{Q} \in \mathcal{Q}_{M}}\left\{-E_{Q_{M}}\left[\sum_{n \leq M} X_{n}\right]-\sum_{n \geq M+1} E_{Q_{n}} X_{n}-\gamma_{\mathcal{A}}(\mathbf{Q})\right\},
$$

where $\mathcal{Q}_{M} \triangleq\left\{\left(Q_{M}, Q_{M+1}, \ldots, Q_{N}\right)\left|Q_{n} \in \mathcal{P}_{n}, Q_{n}\right|_{\mathcal{F}_{M}} \equiv Q_{M} \forall n \geq M\right\}$ and

$$
\gamma_{\mathcal{A}}(\mathbf{Q}) \triangleq \sup _{\mathbf{Y} \in \mathcal{A}}\left\{-E_{Q_{M}}\left[\sum_{n \leq M} Y_{n}\right]-\sum_{n \geq M+1} E_{Q_{n}} Y_{n}\right\} .
$$

Proof. Under the hypotheses of the Proposition and due to the particular form of $\mathcal{C}_{M}$, Assumptions A to D are satisfied. Since $\rho_{\mathcal{A}, M}$ is $\tau$-lower semicontinuous, we can apply Proposition 4.12 in order to obtain representation (4.12). Below we refer to the notation introduced in that Proposition. Since $\mathcal{C}_{M}$ is a positive cone containing 0, then by Proposition 3.11 the term $\gamma_{\mathcal{C}}$ vanishes on $\mathcal{B}_{\mathcal{C}}$. If $(\mathbf{c}, \mathbf{Q}) \in \mathcal{B}_{\mathcal{C}}$, then $c_{n}=1$ for all $n$. Indeed, fix $n$ and observe that $\pm \mathbf{e}_{n} \in \mathcal{C}_{M}$. Therefore $\pm c_{n} \mp 1 \leq 0$, i.e. $c_{n}=1$. Next we prove that if $(\mathbf{1}, \mathbf{Q}) \in \mathcal{B}_{\mathcal{C}}$, then for any $n, Q_{n} \equiv Q_{M}$ on $\mathcal{F}_{n \wedge M}$. Indeed, fix $n$ and $A \in \mathcal{F}_{n \wedge M}$ and observe that the two processes $\pm I_{A} \mathbf{e}_{n} \mp I_{A} \mathbf{e}_{M}$ belong to $\mathcal{C}_{M}$, since $I_{A}$ is $\mathcal{F}_{M}$-measurable and the final cumulated sum vanishes in both cases. Therefore $\pm Q_{n}(A) \mp Q_{M}(A) \leq 0$, so that $Q_{n}(A)=Q_{M}(A)$. Finally, we obtain (5.1) by noting that if $n \leq M$ and $Q_{n} \equiv Q_{M}$ on $\mathcal{F}_{n}$, then $E_{Q_{n}} X=E_{Q_{M}} X$ for any $X \in L_{n}^{\infty}$.

Remark 5.2 When $M=N$, i.e. when there is no constraint on the timing of choice, then $\mathcal{C}_{N}=\mathcal{L}_{\Sigma}^{\infty}$ and $(5.1)$ becomes:

$$
\rho_{\mathcal{A}, N}(\mathbf{X})=\rho_{\mathcal{A}}(\mathbf{X})=\sup _{Q \ll P}\left\{-E_{Q}\left[\sum_{n=1}^{N} X_{n}\right]-\gamma_{\mathcal{A}}(Q)\right\},
$$

where

$$
\gamma_{\mathcal{A}}(\mathbf{Q}) \triangleq \sup _{\mathbf{Y} \in \mathcal{A}}\left\{-E_{Q}\left[\sum_{n=1}^{M} Y_{n}\right]\right\} .
$$

In other words, $\rho_{\mathcal{A}}(\mathbf{X})=\rho_{\text {static }}\left(\sum_{n=1}^{N} X_{n}\right)$, where $\rho_{\text {static }}$ is a suitable static risk measure. In particular, the set $\mathcal{A}_{\text {static }} \subset L^{\infty}$ of random variables

$$
\mathcal{A}_{\text {static }}=\left\{\sum_{n=1}^{N} X_{n} \mid \mathbf{X} \in \mathcal{A}\right\}
$$


is an acceptability set of $\rho_{\text {static }}$.

\section{A credit constraint}

Here, we discuss another type of constraint. The regulator may impose a bound on the loans that the agent can take out. If $\beta \in[0,+\infty]$ is such a bound (or credit line), the corresponding set of permitted strategies is:

$$
\mathcal{C}=\mathcal{C}^{\beta} \triangleq\left\{\mathbf{Y} \in \mathcal{L}_{\Sigma}^{\infty} \mid \sum_{n \geq m} Y_{n} \geq-\beta \forall m=1, \ldots, N\right\} .
$$

If $\beta=+\infty$ then no credit line constraints are considered and $\mathcal{C}^{\infty}=\mathcal{L}_{\Sigma}^{\infty}$. Otherwise, the regulator is requiring that, at any $n=1, \ldots, N$, the portfolio value $V_{n}$ is greater than $-\beta$ and observe now that $V_{n}=V_{0}-\sum_{i \leq n} Y_{i}=\sum_{i=1}^{N} Y_{i}-\sum_{i \leq n} Y_{i}=\sum_{i \geq n+1} Y_{i}$.

We provide here the dual representation for this class of capital requirements, setting $\rho_{\mathcal{A}}^{\beta}=\rho_{\mathcal{A}, \mathcal{C}^{\beta}}$, where $\mathcal{A}$ is an acceptability set.

Proposition 6.1 Let $\beta \in[0,+\infty)$. Assume that $\mathcal{A}$ is convex, monotone and satisfies assumption $B$ and that $\rho_{\mathcal{A}}^{\beta}$ is $\tau$-lower semicontinuous. Then (4.12) holds and

$$
\mathcal{B}_{\mathcal{C}} \subset\left\{(\mathbf{c}, \mathbf{Q}) \in \mathcal{B} \mid c_{1} \leq 1,\left(c_{1} \frac{d Q_{1}}{d P}, \ldots, c_{N} \frac{d Q_{N}}{d P}\right) \text { is a P-supermartingale }\right\} .
$$

When $\beta=0$, the term $\gamma_{\mathcal{C}}$ vanishes in $\mathcal{B}_{\mathcal{C}}$.

Proof. The validity of (4.12) is guaranteed by the hypotheses as in the previous proposition. We now prove that for any $\beta \in[0,+\infty)$ :

$$
0^{+} \mathcal{C}^{\beta}=\left\{\mathbf{Y} \in \mathcal{L}_{\Sigma}^{\infty} \mid \sum_{n \geq m} Y_{n} \geq 0 \forall m=1, \ldots, N\right\}
$$

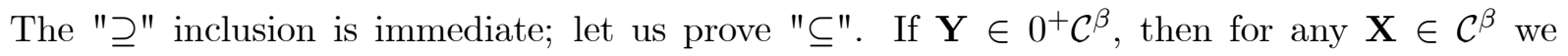
have $\mathbf{X}+\mathbf{Y} \in \mathcal{C}^{\beta}$. Since $\mathbf{0} \in \mathcal{C}^{\beta}$ we have $0^{+} \mathcal{C}^{\beta} \subseteq \mathcal{C}^{\beta} \subset \mathcal{L}_{\Sigma}^{\infty}$. If $\mathbf{Y} \in 0^{+} \mathcal{C}^{\beta}$, then by definition $\mathbf{0}+\lambda \mathbf{Y} \in \mathcal{C}^{\beta}$ for all $\lambda \geq 0$ so that for any $m=1, \ldots, N$, it holds

$$
\lambda \sum_{n \geq m} Y_{n} \geq-\beta \quad \forall \lambda \geq 0,
$$

which readily yields $\sum_{n \geq m} Y_{n} \geq 0$.

Let now $(\mathbf{c}, \mathbf{Q}) \in \mathcal{B}_{\mathcal{C}}$. Since $\mathbf{e}^{1} \in 0^{+} \mathcal{C}^{\beta}$, it must hold $c_{1}-1 \leq 0$, i.e. $c_{1} \leq 1$. Fix now $n \leq N-1$ and $A \in \mathcal{F}_{n}$ and define $\mathbf{Y}=-I_{A} \mathbf{e}^{n}+I_{A} \mathbf{e}^{n+1}$ which clearly belongs to $0^{+} \mathcal{C}^{\beta}$. Therefore it must hold $-c_{n} Q_{n}(A)+c_{n+1} Q_{n+1}(A) \leq 0$. Setting $Z_{n} \triangleq c_{n} \frac{d Q_{n}}{d P}$, we can conclude that for any $n \leq N-1$ :

$$
E_{P} Z_{n} I_{A} \geq E_{P} Z_{n+1} I_{A} \quad \forall A \in \mathcal{F}_{n}
$$

which is equivalent to say that $Z_{n} \geq E_{P}\left(Z_{n+1} \mid \mathcal{F}_{n}\right)$, i.e. the process $\mathbf{Z}$ is a $P$-supermartingale. Finally, if $\beta=0$, then $0^{+} \mathcal{C}^{\beta}$ is a positive cone containing $\mathbf{0}$ and we recover that $\gamma_{\mathcal{C}}$ vanishes on $\mathcal{B}_{\mathcal{C}}$ by Proposition 3.11.

Remark 6.2 Note that, as a consequence of the supermartingale property, two necessary conditions for a couple (c, $\mathbf{Q})$ to enter the representation (4.12) in the case of credit line are: 
1. $\left(c_{n}\right)_{n=1}^{N}$ is a decreasing sequence. This broadly means that quantities which are liquidated at the first dates matter more than those liquidated at the end, for the sake of risk assessment.

2. If $c_{n}>0, Q_{n}(A) \geq d_{n} Q_{n+1}(A)$ for any $A \in \mathcal{F}_{n}$, where $d_{n} \triangleq \frac{c_{n+1}}{c_{n}} \leq 1$ is independent from $A$. In particular, if $c_{n}=c_{n+1}$, then $Q_{n+1} \equiv Q_{n}$ on $\mathcal{F}_{n}$.

\section{Some examples}

We end this paper by considering, in the simplified setting of one intermediate date, namely $N=2$, some simple, instructive examples of acceptance sets for processes and related capital requirements. We concentrate here on two relevant classes of acceptance sets: those which can be written as a product of acceptance sets for random variables, and those which are naturally defined, as in the static setting, on the basis of a time-additive utility for processes.

\subsection{Product-type acceptance sets}

Assume that $\mathcal{A}_{1} \subset L_{1}^{\infty}$ and $\mathcal{A}_{2} \subset L_{2}^{\infty}$ are two acceptance sets of random variables and define $\mathcal{A} \triangleq \mathcal{A}_{1} \times \mathcal{A}_{2} \subset \mathcal{L}^{\infty}$. Remind that $\rho_{\mathcal{A}}, \rho_{\mathcal{A}, 0}$ and $\rho_{\mathcal{A}}^{\beta}(\beta \geq 0)$ are the capital requirements associated to the sets of permitted strategies $\mathcal{C}=\mathcal{L}_{\Sigma}^{\infty}, \mathcal{C}_{0}$ and $\mathcal{C}^{\beta}$, respectively. Denote with $\rho_{1}$ and $\rho_{2}$ the static capital requirements associated to $\mathcal{A}_{1}$ and $\mathcal{A}_{2}$.

Proposition 7.1 Let $\mathcal{A}=\mathcal{A}_{1} \times \mathcal{A}_{2}$, where $\mathcal{A}_{1} \subset L_{1}^{\infty}$ and $\mathcal{A}_{2} \subset L_{2}^{\infty}$ are monotone and satisfy (2.2). Then $\rho_{\mathcal{A}}, \rho_{\mathcal{A}, 0}$ and $\rho_{\mathcal{A}}^{\beta}$ are finite valued and monotone; moreover we can write

$$
\begin{aligned}
& \rho_{\mathcal{A}}\left(X_{1}, X_{2}\right)=\inf \left\{\rho_{2}\left(X_{1}+X_{2}-Y\right) \mid Y \in \mathcal{A}_{1}\right\} \\
& \rho_{\mathcal{A}, 0}\left(X_{1}, X_{2}\right)=\rho_{1}\left(X_{1}\right)+\rho_{2}\left(X_{2}\right) \\
& \rho_{\mathcal{A}}^{\beta}\left(X_{1}, X_{2}\right)=\max \left[-\beta,-\beta+\inf _{Y \in \mathcal{A}_{1}}\left(\operatorname{ess} . i n f\left(Y-X_{1}\right)\right), \rho_{\mathcal{A}}\left(X_{1}, X_{2}\right)\right] .
\end{aligned}
$$

Proof. Assumption $\mathrm{C}$ is trivially satisfied in each of the three choices of $\mathcal{C}$, while assumptions $\mathrm{A}$ and $\mathrm{B}$ follow from the hypotheses we have made on $\mathcal{A}_{1}$ and $\mathcal{A}_{2}$. The first part of the Proposition is then a direct consequence of Propositions 4.5 and 4.6. Concerning the explicit representations, we have

$$
\begin{aligned}
\rho_{\mathcal{A}}\left(X_{1}, X_{2}\right) & =\inf \left\{m \in \mathbb{R} \mid \exists Z \in L_{1}^{\infty} \text { s.t. } X_{1}+Z \in \mathcal{A}_{1}, X_{2}+m-Z \in \mathcal{A}_{2}\right\} \\
& =\inf \left\{m \in \mathbb{R} \mid \exists Y \in \mathcal{A}_{1} \text { s.t. } X_{2}+m-\left(Y-X_{1}\right) \in \mathcal{A}_{2}\right\} \\
& =\inf _{Y \in \mathcal{A}_{1}} \inf \left\{m \in \mathbb{R} \mid X_{2}+X_{1}-Y+m \in \mathcal{A}_{2}\right\} \\
& =\inf _{Y \in \mathcal{A}_{1}} \rho_{2}\left(X_{1}+X_{2}-Y\right) .
\end{aligned}
$$

The other two representations are similarly proved.

The simplest example of product-type acceptance set is

$$
\mathcal{A}=\mathcal{L}_{+}^{\infty} \triangleq\left\{\left(X_{1}, X_{2}\right) \in \mathcal{L}^{\infty} \mid X_{1}, X_{2} \geq 0\right\}
$$


The hypotheses of the previous proposition are met and, after some standard calculation, we have:

$$
\begin{aligned}
& \rho_{\mathcal{L}_{+}^{\infty}}\left(X_{1}, X_{2}\right)=-\operatorname{ess} . i n f\left(X_{1}+X_{2}\right) \\
& \rho_{\mathcal{L}_{+}^{\infty}, 0}\left(X_{1}, X_{2}\right)=-\operatorname{ess} . i n f\left(X_{1}\right)-\operatorname{ess} . i n f\left(X_{2}\right) \\
& \rho_{\mathcal{L}_{+}^{\infty}}^{\beta}\left(X_{1}, X_{2}\right)=\max \left[-\beta,-\beta-\operatorname{ess} . i n f\left(X_{1}\right),-\operatorname{ess} . i n f\left(X_{1}+X_{2}\right)\right] .
\end{aligned}
$$

\subsection{Utility-based acceptance sets}

This class of examples generalizes utility-based static risk measures as defined in Section 2.4. Assume that the agent's preferences over payoff streams are described by a time-additive expected utility functional:

$$
U\left(X_{1}, X_{2}\right)=E_{P} u\left(X_{1}\right)+b E_{P} u\left(X_{2}\right),
$$

where $u: \mathbb{R} \rightarrow \mathbb{R}$ is an utility function and $b>0$. A natural acceptance set of processes is then

$$
\mathcal{A}=\left\{\left(X_{1}, X_{2}\right) \in \mathcal{L}^{\infty} \mid U\left(X_{1}, X_{2}\right) \geq U\left(X_{1}^{*}, X_{2}^{*}\right)\right\}
$$

where $\left(X_{1}^{*}, X_{2}^{*}\right)$ is a reference process. If we choose the exponential utility function $u_{\gamma}(x)=$ $1-\exp (-\gamma x), \gamma>0$, and $\left(X_{1}^{*}, X_{2}^{*}\right)=(0,0)$, then we obtain, after standard calculations

$$
\mathcal{A}=\left\{\left(X_{1}, X_{2}\right) \in \mathcal{L}^{\infty} \mid E_{P} e^{-\gamma X_{1}}+b E_{P} e^{-\gamma X_{2}} \leq 1+b\right\} .
$$

Clearly, this is not a product-type acceptance set, so that we cannot apply Proposition 7.1. Moreover, assumption B is not satisfied for any choice of $\gamma$ and $b$. However, we show below that choosing $\mathcal{C}=\mathcal{C}_{0}$ or $\mathcal{C}_{0}^{\beta}=\mathcal{C}_{0} \cap \mathcal{C}^{\beta}$, the resulting capital requirement is finite valued and admits an explicit formulation. Let $\rho_{\gamma}$ the (static) entropic risk measure as defined in section 2, i.e.

$$
\rho_{\gamma}(X)=\frac{1}{\gamma} \log E_{P} e^{-\gamma X}
$$

Proposition 7.2 Let $\mathcal{A}$ be defined as in (7.2). For any fixed $b, \gamma>0$ and $\left(X_{1}, X_{2}\right) \in \mathcal{L}^{\infty}$ it holds

$$
\rho_{\mathcal{A}, 0}\left(X_{1}, X_{2}\right)=\rho_{\gamma}\left(X_{1}\right)+\rho_{\gamma}\left(X_{2}\right)-\frac{1}{\gamma} \log \frac{(1+b)^{2}}{4 b}
$$

Moreover, for any $\beta \geq 0$,

$$
\rho_{\mathcal{A}, 0}^{\beta}\left(X_{1}, X_{2}\right)=\max \left[-\beta, \rho_{0, \mathcal{A}}\left(X_{1}, X_{2}\right)\right],
$$

where $\rho_{\mathcal{A}, 0}^{\beta}$ is the capital requirement associated to $\mathcal{C}_{0}^{\beta}=\mathcal{C}_{0} \cap \mathcal{C}^{\beta}$.

Proof. For fixed $b, \gamma, X_{1}, X_{2}$, consider the following inequalities, where $m, \alpha, x \in \mathbb{R}$ :

$$
\begin{array}{rl}
(*)_{m, \alpha} & E_{P} e^{-\gamma\left(X_{1}+\alpha\right)}+b E_{P} e^{-\gamma\left(X_{2}+m-\alpha\right)} \leq 1+b, \\
(* *)_{m, x} & b e^{-\gamma m} E_{P} e^{-\gamma X_{2}} x^{2}-(1+b) x+E_{P} e^{-\gamma X_{1}} \leq 0,
\end{array}
$$

Putting $x=e^{\gamma \alpha}$ a standard computation shows that, for any fixed $m \in \mathbb{R},\left({ }^{*}\right)_{m, \alpha}$ is satisfied for some $\alpha$ if and only if $(* *)_{m, x}$ is satisfied for some $x>0$, i.e. the equation:

$$
b e^{-\gamma m} E_{P} e^{-\gamma X_{2}} x^{2}-(1+b) x+E_{P} e^{-\gamma X_{1}}=0
$$


has two distinct solutions $x_{1}<x_{2}$ and $x_{2}>0$. The first condition is satisfied exactly when the discriminant is strictly positive, i.e. when

$$
0<\Delta=(1+b)^{2}-4 b E_{P} e^{-\gamma X_{2}} E_{P} e^{-\gamma X_{1}} e^{-\gamma m},
$$

which is equivalent to

$$
m>\rho_{\gamma}\left(X_{1}\right)+\rho_{\gamma}\left(X_{2}\right)-\frac{1}{\gamma} \log \frac{(1+b)^{2}}{4 b} .
$$

The signs of the coefficients of the equation (7.5) guarantee that both solutions are positive. Then we have:

$$
\begin{aligned}
\rho_{\mathcal{A}, 0}\left(X_{1}, X_{2}\right) & \triangleq \inf \left\{m \in \mathbb{R} \mid \exists \alpha \in \mathbb{R} \text { s.t. }\left({ }^{*}\right)_{m, \alpha} \text { is satisfied }\right\} \\
& =\rho_{\gamma}\left(X_{1}\right)+\rho_{\gamma}\left(X_{2}\right)-\frac{1}{\gamma} \log \frac{(1+b)^{2}}{4 b} .
\end{aligned}
$$

For any $\beta \geq 0$ we have, by definition:

$$
\rho_{\mathcal{A}, 0}^{\beta}\left(X_{1}, X_{2}\right)=\inf \left\{m \geq-\beta \in \mathbb{R} \mid \exists \alpha \in \mathbb{R}, \alpha \leq m+\beta \text { s.t. }\left({ }^{*}\right)_{m, \alpha} \text { is satisfied }\right\}
$$

In addition to (7.6), in this case we are requiring also that $x_{1}<e^{\gamma(m+\beta)}$. However a lengthy, but standard calculation shows that this requirement is satisfied under (7.6). Therefore, (7.4) follows immediately from (7.7).

Remark 7.3 Since $\rho_{\gamma}$ admits the dual representation (2.4), we obtain

$$
\rho_{\mathcal{A}, 0}\left(X_{1}, X_{2}\right)=\sup _{Q_{1}, Q_{2} \ll P}\left\{-E_{Q_{1}}\left[X_{1}\right]-E_{Q_{2}}\left[X_{2}\right]-F_{b, \gamma}\left(Q_{1}, Q_{2}\right)\right\}
$$

where

$$
F_{b, \gamma}\left(Q_{1}, Q_{2}\right) \triangleq \frac{1}{\gamma}\left\{\left(H\left(Q_{1} \mid P\right)+H\left(Q_{2} \mid P\right)-\log \frac{(1+b)^{2}}{4 b}\right\} \in(-\infty,+\infty] .\right.
$$

The parameter $b$ enters the explicit formulation of $\rho_{\mathcal{A}, 0}$ only through the additive term $\frac{1}{\gamma} g(b)$ where

$$
g(b)=\log \frac{(1+b)^{2}}{4 b}, b>0 .
$$

Observe that $g\left(b^{-1}\right)=g(b), g \geq 0, g(1)=0, g$ has a minimim in $b=1$

As in (2.5), we have:

$$
\lim _{\gamma \rightarrow \infty} \rho_{\mathcal{A}, 0}\left(X_{1}, X_{2}\right)=\sup _{Q_{i} \ll P: H\left(Q_{i} \mid P\right)<\infty}\left\{-E_{Q_{1}}\left[X_{1}\right]-E_{Q_{2}}\left[X_{2}\right]\right\}
$$

\subsection{Comparison of simple binomial trees}

In this final section we show that, by appropriately selecting the sets $\mathcal{C}$ and/or $\mathcal{A}$ it is possible to point out some of the different characteristics of risk processes. Consider the following five simple trees, assuming that the probability of each leaf is $1 / 4$. The amounts 1 and -1 are expressed in some monetary units and are already discounted (so that we may assume a zero interest rate). 

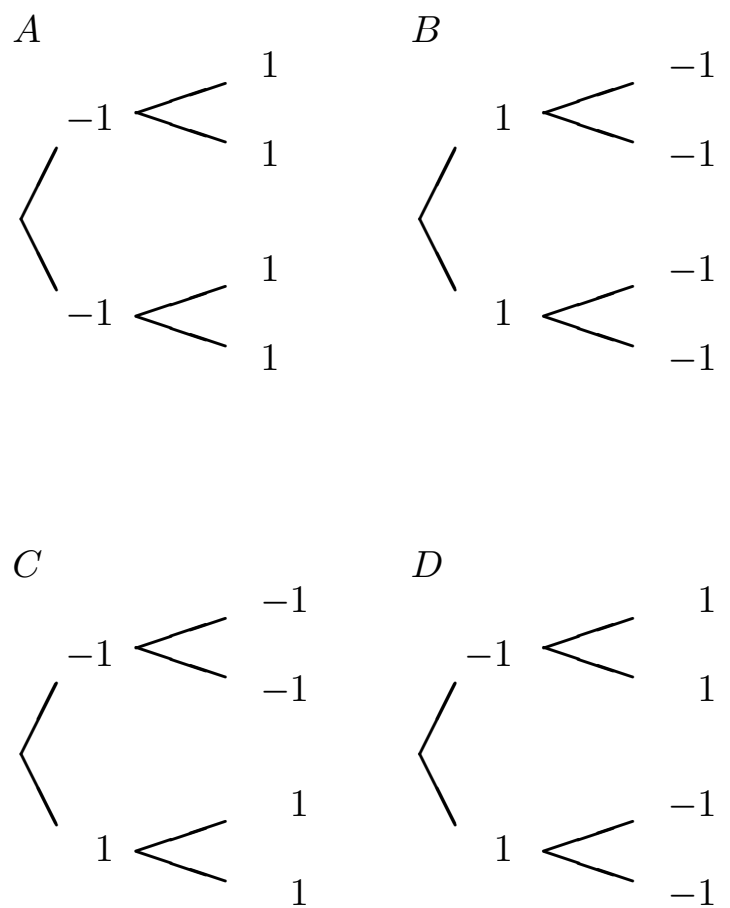

D

E
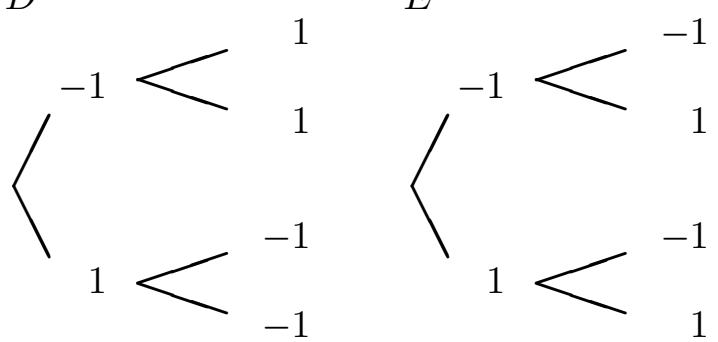

Observe that the trees $\mathrm{A}$ and $\mathrm{B}$ are deterministic and that $\mathrm{C}, \mathrm{D}, \mathrm{E}$ have the same marginal distributions, but the uncertainty resolves at time $t_{1}$ in $\mathrm{C}$ and $\mathrm{D}$ and at time $t_{2}$ in E. Moreover, the expected cumulated final payoff, i.e. $E_{P}\left[X_{1}+X_{2}\right]$, vanishes for all five trees.

Let $\mathcal{A} \triangleq \mathcal{L}_{+}^{\infty}$ and

$$
\mathcal{A}^{\prime} \triangleq\left\{\left(X_{1}, X_{2}\right) \mid E_{P}\left[X_{1}\right] \geq 0 \text { and } E_{P}\left[X_{2} \mid \mathcal{F}_{1}\right] \geq 0\right\}
$$

Observe that $\mathcal{A} \subset \mathcal{A}^{\prime}$.

We easily compute the following values for the capital requirements associated to the above five trees:

\begin{tabular}{|c|c|c|c|c|c|c|c|}
\hline & $\rho_{\mathcal{A}}$ & $\rho_{\mathcal{A}, 0}$ & $\rho_{\mathcal{A}}^{0}$ & $\rho_{\mathcal{A}}^{0.5}$ & $\rho_{\mathcal{A}}^{\beta}, \beta \geq 1$ & $\rho_{\mathcal{A}^{\prime}}$ & $\rho_{\mathcal{A}^{\prime}, 0}$ \\
\hline $\mathrm{A}$ & 0 & 0 & 1 & 0.5 & 0 & 0 & 0 \\
\hline $\mathrm{B}$ & 0 & 0 & 0 & 0 & 0 & 0 & 0 \\
\hline $\mathrm{C}$ & 2 & 2 & 2 & 2 & 2 & 0 & 1 \\
\hline $\mathrm{D}$ & 0 & 2 & 1 & 0.5 & 0 & 0 & 1 \\
\hline $\mathrm{E}$ & 2 & 2 & 2 & 2 & 2 & 0 & 0 \\
\hline
\end{tabular}

Some comments about these numerical results are in order:

1. The trees A and B differ only for the order of the payoffs: in A we have a pay-out followed by a pay-in of the same amount, while the reversed flow is described by B. If these fixed amounts (pay-out and pay-in) are large, for instance comparable to the total wealth of the agent, then the tree $\mathrm{A}$ should be considered riskier than B. Note that, within the examples we are considering, this is true only for $\rho_{\mathcal{A}}^{\beta}$ when $\beta=0$ or 0.5 , i.e. when we introduce a sufficiently strict credit line. If $\beta \geq 1$ the corresponding capital requirement is not able to distinguish between $\mathrm{A}$ and $\mathrm{B}$. 
2. Observe that $\mathrm{C}, \mathrm{D}$ and $\mathrm{E}$, which are not deterministic, are generally riskier than $\mathrm{A}$ and $\mathrm{B}$.

3. Note the different evaluation of the tree $\mathrm{D}$ given by $\rho_{\mathcal{A}}$ and $\rho_{\mathcal{A}, 0} ; \rho_{\mathcal{A}}$ is lower since it is possible to hedge efficiently (with random variables) the payoffs, while only a constant hedge is permitted when computing $\rho_{\mathcal{A}, 0}$.

4. Looking at the trees $\mathrm{C}$ and $\mathrm{E}$, we see that they both have the same "worst scenario" constituted by the the upper trajectory $(-1,-1)$. When $\mathcal{A} \triangleq \mathcal{L}_{+}^{\infty}$ then each risk measure $\rho_{\mathcal{A}}, \rho_{\mathcal{A}, 0}, \rho_{\mathcal{A}, 0}^{\beta}$ gives the same evaluation: - ess.inf $\left(X_{1}+X_{2}\right)=-\operatorname{ess} . i n f\left(X_{1}\right)-\operatorname{ess} . i n f\left(X_{2}\right)=$ 2. However, if we consider the risk measure $\rho_{\mathcal{A}^{\prime}, 0}$ then it is possible to distinguish between $\mathrm{C}$ and E.

Next we compute, for some choices of $(\gamma, b)$, the capital requirement $\rho_{\mathcal{A}, 0}$ when $\mathcal{A}$ is the utility based acceptance set defined in (7.2). Note that for any $(\gamma, b), \rho_{\mathcal{A}, 0}$ assigns, by (7.3), the same value to $\mathrm{A}$ and $\mathrm{B}$ and, separately, to $\mathrm{C}, \mathrm{D}$ and $\mathrm{E}$. The following table resumes some computations for the trees $\mathrm{C}, \mathrm{D}$ and $\mathrm{E}$ for different values of $b$ (rows) and $\gamma$ (columns).

\begin{tabular}{|c|c|c|c|c|c|}
\hline & $\gamma=0.5$ & $\gamma=1$ & $\gamma=2$ & $\gamma=10$ & $\gamma \rightarrow \infty$ \\
\hline$b=0.50$ & 0.245 & 0.750 & 1.266 & 1.850 & 2 \\
\hline$b=0.75$ & 0.439 & 0.847 & 1.315 & 1.859 & 2 \\
\hline$b=1$ & 0.480 & 0.868 & 1.325 & 1.861 & 2 \\
\hline
\end{tabular}

The dependence on $b$ (for a fixed $\gamma$ ) is simple since it is described by $g$ (see remark 7.3). In the last column we evaluate $\lim _{\gamma \rightarrow \infty} \rho_{\mathcal{A}, 0}\left(X_{1}, X_{2}\right)$. Since we are in a finite probability space, from the equation (7.8) we deduce that

$$
\lim _{\gamma \rightarrow \infty} \rho_{\mathcal{A}, 0}\left(X_{1}, X_{2}\right) \rightarrow-\operatorname{ess} . i n f\left(X_{1}\right)-\operatorname{ess} . i n f\left(X_{2}\right)=\rho_{\mathcal{L}_{+}^{\infty}, 0}\left(X_{1}, X_{2}\right),
$$

for any process $\left(X_{1}, X_{2}\right)$.

\section{References}

Artzner, P., F. Delbaen, J.M. Eber, and D. Heath (1999): Coherent Measures of Risk, Math. Finance 9(3), 203-228

Artzner, P., F. Delbaen, J.M Eber, D. Heath, and H. Ku (2004): Coherent Multiperiod Risk Adjusted Values and Bellman's Principle, Working Paper, ETH Zürich (first version 2002)

Barrieu P. AND El Karoui N. (2005): Inf-convolution of risk measures and optimal risk transfer, Finance Stoch. 9(2), 269-298.

Brachinger, H.W., and M. Weber (1997): Risk as a Primitive: a Survey of Measures of Perceived Risk, OR-Spektrum 19(3), 235-250

Carr, P., H. Geman, and D.B. Madan (2001): Pricing and Hedging in Incomplete Markets, J. Financial Econ. 62, 131-167

Cheridito, P., F. Delbaen, and M. Kupper (2004a): Coherent and Convex Risk Measures for Bounded Càdlàg Processes, Stoch. Proc. Appl. 112(1), 1-22 
Cheridito, P., F. Delbaen, and M. Kupper (2004b): Coherent and Convex Risk Measures for Unbounded Càdlàg Processes, Working Paper, ETH Zürich, to appear in Finance Stoch.

De Finetti, B. (1931): Sul Concetto di Media, Giornale degli Economisti 2

Delbaen, F. (2002): Coherent Measures of Risk on General Probability Spaces; in Advances in Finance and Stochastics, K. Sandmann and P.J. Schönbucher eds. Berlin: Springer-Verlag

Föllmer, H., and A. Schied (2002): Convex Measures of Risk and Trading Constraints, Finance Stoch. 6(4) 429-447

Föllmer, H., and A. Schied (2004): Stochastic Finance. An Introduction in Discrete Time, 2nd Edition, Berlin: de Gruyter

Frittelli, M., and E. Rosazza Gianin (2002): Putting Order in Risk Measures, J. of Banking and Finance 26, 1473-1486

Frittelli, M., and E. Rosazza Gianin(2004): Dynamic Convex Risk Measures; in Risk Measures for the 21st Century, G. Szego, ed. New York: Wiley

Kreps, D.M., and E.L. Porteus (1978): Temporal Resolution of Uncertainty and Dynamic Choice Theory, Econometrica 46, 185-200

Pflug, G., Ruszczynski A. (2001): A Risk Measure for Income Streams, Working Paper, University of Vienna

Riedel, F. (2004): Dynamic Coherent Risk Measures, Stoch. Proc. Appl. 112, 185-200

Rockafellar, R.T. (1970): Convex Analysis, Princeton: Princeton University Press

Scandolo, G. (2003): Risk Measures in a Dynamic Setting, PhD Thesis, Università di Milano

Scandolo, G. (2004): Models of Capital Requirements in Static and Dynamic Settings, Economic Notes 33, 415-435

Staum, J. (2004): Fundamental Theorems of Asset Pricing for Good Deal Bounds, Math. Finance 14, 141-161

Weber, S. (2003): Distribution-invariant Dynamic Risk Measures, Working Paper, Humboldt Universität Berlin

Wang, T. (1999) A Class of Dynamic Risk Measures, Working Paper, University of British Columbia 